\title{
Asymptotics of the solutions of the stochastic lattice wave equation
}

\author{
Tomasz Komorowski* $\quad$ Stefano Olla ${ }^{\dagger} \quad$ Lenya Ryzhik ${ }^{\ddagger}$
}

January 2, 2018

\begin{abstract}
We consider the long time limit for the solutions of a discrete wave equation with a weak stochastic forcing. The multiplicative noise conserves the energy, and in the unpinned case also conserves the momentum. We obtain a time-inhomogeneous Ornstein-Uhlenbeck equation for the limit wave function that holds both for square integrable and statistically homogeneous initial data. The limit is understood in the point-wise sense in the former case, and in the weak sense in the latter. On the other hand, the weak limit for square integrable initial data is deterministic.
\end{abstract}

\section{Introduction}

Energy transport and dispersion in dynamics of oscillators in a lattice have been investigated in many situations in order to understand macroscopic thermal conductivity properties. A typical example is the Fermi-Pasta-Ulam chain under the Hamiltonian evolution corresponding to a quartic interaction potential. In the one dimension the Hamiltonian of the closed system of length $N$ with periodic boundary conditions is given by

$$
\mathcal{H}=\sum_{y \in \mathbb{Z} / N \mathbb{Z}}\left(\frac{\mathfrak{p}_{y}^{2}}{2 m}+\frac{1}{2} \omega_{0}^{2} \mathfrak{q}_{y}^{2}\right)+\sum_{y \in \mathbb{Z} / N \mathbb{Z}}\left[\frac{1}{2}\left(\mathfrak{q}_{y}-\mathfrak{q}_{y-1}\right)^{2}+\gamma\left(\mathfrak{q}_{y}-\mathfrak{q}_{y-1}\right)^{4}\right]
$$

Here $\mathbb{Z} / N \mathbb{Z}$ denotes the group $\{0, \ldots, N-1\}$ with the addition modulo $N, \mathfrak{q}_{y}$ is the displacement of the $y$-th particle from its equilibrium position, $\mathfrak{p}_{y}$ is its momentum and $m$ is the mass. When $\omega_{0} \neq 0$, the particle is confined, this breaks translation invariance, and correspondingly the conservation of the total momentum, and we say that the chain is pinned.

When $\gamma=0$ the Hamiltonian dynamics is given by the discrete in space linear wave equation, and the energy evolution is purely ballistic and dispersive. If $\gamma>0$ and $\omega_{0} \neq 0$, due to the presence of the non-linearity, wave scattering is expected that in turn gives a finite thermal conductivity and consequently a diffusive macroscopic evolution of the energy. If the

\footnotetext{
${ }^{*}$ Institute of Mathematics, UMCS, pl. Marii Curie-Skłodowskiej 1, 20-031, Lublin and IMPAN, ul. Śniadeckich 8, 00-956 Warsaw, Poland, e-mail: komorow@hektor.umcs.lublin.pl

${ }^{\dagger}$ Ceremade, UMR-CNRS 7534, Université Paris Dauphine, Place Marechal Lattre de Tassigny, 75775, Paris Cedex 16, France, e-mail: olla@ceremade.dauphine.fr

${ }^{\ddagger}$ Department of Mathematics, Stanford University, Stanford, CA 94305, USA, e-mail: ryzhik@math.stanford.edu
} 
chain is unpinned, $\omega_{0}=0$, and $\gamma>0$, long waves scatter rarely, giving rise to a superdiffusive behavior of the energy [14].

The mathematical analysis of the macroscopic behavior of the energy is difficult in the case of deterministic nonlinear dynamics, and recently various models considering stochastic perturbations of the dynamics have been proposed. Such perturbations generate scattering qualitatively similar to the one due to the nonlinearity.

In order to mimic the nonlinear dynamics, a noisy perturbation we wish to consider should conserve energy and be local in space [5]. In the unpinned case it is also important that it conserve the momentum, see [2, 3]. The perturbations considered in these papers are given by a random exchange of momentum so that the total kinetic energy is constant (consequently, the total energy is preserved as well, since the position components are untouched by the noise) and the total momentum is also conserved. This is achieved by adding, to each triple of adjacent particles, a diffusion on the corresponding surface of constant energy and momentum. Another example of a noisy perturbation having similar properties appears in a discontinuous in time model in which momenta of pairs of adjacent particles are exchanged at independent random times that are exponentially distributed.

When the interaction is linear, the thermal diffusivity of the energy in these models can be explicitly computed - it is finite for the pinned model but diverges with the size of the system in the unpinned case (corresponding to superdiffusive energy transport for the unpinned model).

The limit dynamics for the spectral measure of the energy in these stochastic models is investigated in [4, where the noise is also rescaled in such a way that there are only finitely many wave collisions in the unit macroscopic time. In a sense, this weak noise limit is similar to the regime where phonon-Boltzmann equation is valid in weakly nonlinear models (cf. [18]). The dynamics is defined in the following way. Consider the infinite lattice $\mathbb{Z}$ with the Hamiltonian associated to the linear evolution (1.1) $(\gamma=0)$, with $N=\infty$, perturbed by a conservative noise. Formally, it is given by the solution of the stochastic differential equations:

$$
\begin{aligned}
\dot{\mathfrak{q}}_{y}(t) & =\mathfrak{p}_{y}(t) \\
d \mathfrak{p}_{y}(t) & =\left(\Delta \mathfrak{q}_{y}-\omega_{0}^{2} \mathfrak{q}_{y}\right) d t+d \eta_{y}(\epsilon t),
\end{aligned}
$$

where $\Delta \mathfrak{q}_{y}=\mathfrak{q}_{y+1}+\mathfrak{q}_{y-1}-2 \mathfrak{q}_{y}$ is the lattice Laplacian. The noise $d \eta_{y}(\epsilon t)$ will be added to model random exchange of momenta between the adjacent sites so that the total kinetic energy and momentum of the system are conserved (see (2.1) for the precise form of the noise). The small parameter $\epsilon>0$ slows down its effect. The total Hamiltonian can be formally written as

$$
\mathcal{H}(\mathfrak{q}, \mathfrak{p})=\sum_{y \in \mathbb{Z}} \frac{\mathfrak{p}_{y}^{2}}{2}+\sum_{x, y \in \mathbb{Z}} \alpha_{x-y} \mathfrak{q}_{x} \mathfrak{q}_{y}
$$

with $\alpha_{0}=\frac{1}{2} \omega_{0}^{2}+1, \alpha_{-1}=\alpha_{1}=-1 / 2$, and $\alpha_{y}=0$ otherwise. The dispersion relation $\omega(k)$ for this system is

$$
\omega(k):=\sqrt{\hat{\alpha}(k)}=\left[\frac{\omega_{0}^{2}}{2}+2 \sin ^{2}(\pi k)\right]^{1 / 2}, \quad k \in \mathbb{T} .
$$

In fact we would admit a broader class of dispersion relations, requiring that $\hat{\alpha}(k)$ is defined as in (2.6) below. Let us introduce the complex wave function

$$
\psi_{y}(t):=(\check{\omega} * \mathfrak{q})_{y}(t)+i \mathfrak{p}_{y}(t)
$$


where $\check{\omega}_{y}$ is the inverse Fourier transform of $\omega(k)$. Its Fourier transform

$$
\hat{\psi}(t, k):=\omega(k) \hat{\mathfrak{q}}(k, t)+i \hat{\mathfrak{p}}(t, k)
$$

satisfies the equation

$$
d \hat{\psi}(t, k)=-i \omega(k) \hat{\psi}(t, k) d t+i d \hat{\eta}(\epsilon t, k)
$$

where $d \hat{\eta}(t, k)$ is the Fourier transform of the noise. Due to the conservation properties of the dynamics, if the initial configuration has finite total energy $\mathcal{H}(\mathfrak{q}(0), \mathfrak{p}(0))<+\infty$, then all the functions introduced in (1.3) and (1.5)-(1.6) are well defined and

$$
\mathcal{H}(\mathfrak{q}(t), \mathfrak{p}(t))=\sum_{y}\left|\psi_{y}(t)\right|^{2}=\int_{\mathbb{T}}|\hat{\psi}(t, k)|^{2} d k
$$

Therefore we can identify $|\hat{\psi}(t, k)|^{2}$ with the energy density in the mode space. In the zero noise case, $|\hat{\psi}(t, k)|^{2}$ is conserved for any $k \in \mathbb{T}$ (i.e. $\partial_{t}|\hat{\psi}(t, k)|^{2}=0$ ). The stochastic conservative perturbation mixes the energies between different modes $k$, and $|\hat{\psi}(t, k)|^{2}$ becomes a random variable. The evolution of the average energy $\mathcal{E}(t, k):=\mathbb{E}|\hat{\psi}(t, k)|^{2}$ was considered in [4]. Since the stochastic perturbation is of order $\epsilon$, to have a visible effect of mixing of different modes we have to look at the time scale $\epsilon^{-1} t$. It was shown in [4] that the limit

$$
\lim _{\epsilon \rightarrow 0} \mathcal{E}\left(\frac{t}{\epsilon}, k\right)=\overline{\mathcal{E}}(t, k)
$$

exists in the sense of distributions, and is the solution of the linear kinetic equation

$$
\partial_{t} \overline{\mathcal{E}}(t, k)=\int_{\mathbb{T}} R\left(k, k^{\prime}\right)\left[\overline{\mathcal{E}}\left(t, k^{\prime}\right)-\overline{\mathcal{E}}(t, k)\right] d k^{\prime}
$$

with the initial condition $\overline{\mathcal{E}}(0, k)=|\hat{\psi}(0, k)|^{2}$. The scattering kernel $R\left(k, k^{\prime}\right)$ is given by (3.2) below.

The goal of the present article is to obtain a direct information on the wave function $\hat{\psi}(t / \epsilon, k)$, as was done in [1] for the Schrödinger equation, and not only for the average energy. It follows from (1.7) that the unperturbed (by noise) evolution of this function is governed by the highly oscillating factor $e^{-i \omega(k) t / \varepsilon}$ (after we rescale the time). It is therefore reasonable to consider, in case of the perturbed system, the compensated wave function of the form

$$
\tilde{\psi}^{(\epsilon)}(t, k):=e^{i \omega(k) t / \varepsilon} \hat{\psi}(t / \varepsilon, k) .
$$

We show that once we compensate for fast oscillations, the wave function converges in law to the solution a Langevin equation driven by (1.9). More precisely, we prove in Theorem 3.1 below, existence of the limit (in law and pointwise in $k$ ):

$$
\lim _{\varepsilon \rightarrow 0} \tilde{\psi}^{(\epsilon)}(t, k)=\tilde{\psi}(t, k)
$$

The limit $\tilde{\psi}(t, k)$ is a complex valued stochastic process satisfying the linear (time inhomogeneous) Ornstein-Uhlenbeck equation

$$
d \tilde{\psi}(t, k)=-\frac{\hat{\beta}(k)}{4} \tilde{\psi}(t, k) d t+\sqrt{\mathcal{R}(t, k)} d w_{k}(t),
$$


with the initial condition $\tilde{\psi}(0, k)=\hat{\psi}(0, k)$. Here

$$
\begin{gathered}
\hat{\beta}(k)=2 \int_{\mathbb{T}} R\left(k, k^{\prime}\right) d k^{\prime} \\
\mathcal{R}(t, k)=\int_{\mathbb{T}} \overline{\mathcal{E}}\left(t, k^{\prime}\right) R\left(k, k^{\prime}\right) d k^{\prime},
\end{gathered}
$$

and $\left\{w_{k}(t)\right\}$ is a family of pairwise independent standard complex valued Brownian motions parametrized by $k \in \mathbb{T}$. That is, they are complex valued, jointly Gaussian, centered processes satisfying

$$
\mathbb{E}\left[w_{k}(t) w_{k^{\prime}}(s)\right]=0 \quad \text { and } \quad \mathbb{E}\left[w_{k^{\prime}}^{*}(t) w_{k}(s)\right]=\delta_{k, k^{\prime}} t \wedge s
$$

for all $t, s \geq 0$ and $k, k^{\prime} \in \mathbb{T}$. Here $\delta_{k, k^{\prime}}=0$ for $k \neq k^{\prime}$ and $\delta_{k, k}=1$. Equation (1.11) has the explicit solution

$$
\tilde{\psi}(t, k)=e^{-\frac{1}{4} \hat{\beta}(k) t} \hat{\psi}(0, k)+\int_{0}^{t} e^{-\frac{1}{4} \hat{\beta}(k)(t-s)} \sqrt{\mathcal{R}(s, k)} d w_{k}(s) .
$$

In particular, we have

$$
\mathbb{E}|\tilde{\psi}(t, k)|^{2}=e^{-\frac{1}{2} \hat{\beta}(k) t}|\hat{\psi}(0, k)|^{2}+\int_{0}^{t} e^{-\frac{1}{2} \hat{\beta}(k)(t-s)} \mathcal{R}(s, k) d s
$$

which is equivalent to (1.9), since $\overline{\mathcal{E}}(t, k)=\mathbb{E}|\tilde{\psi}(t, k)|^{2}$. Initial conditions such that $\int_{\mathbb{T}}|\hat{\psi}(0, k)|^{2} d k<$ $\infty$ correspond to a local perturbation of the zero temperature equilibrium. We are also interested in the macroscopic evolution of the equilibrium states at a positive temperature $T>0$, starting with random data distributed by the Gibbs measure at temperature $T$. In the mode space this is a centered, complex valued, Gaussian random field with distribution valued $\hat{\psi}(k)$. Its covariance is given by

$$
\mathbb{E}\left[\hat{\psi}^{*}(k) \hat{\psi}\left(k^{\prime}\right)\right]=T \delta\left(k-k^{\prime}\right), \quad \mathbb{E}\left[\hat{\psi}(k) \hat{\psi}\left(k^{\prime}\right)\right]=0 .
$$

Here $\delta\left(k-k^{\prime}\right)$ is Dirac's delta function. For any $T$, the corresponding Gibbs measure is invariant under the dynamics, due to the conservation of energy. Actually, in Section 3.2 we consider more general class of space homogeneous Gaussian random initial conditions whose law is not necessarily stationary in time. More precisely, we show (see Theorem 3.3) that if the law of the initial condition is a homogeneous, centered Gaussian field with the covariance given by

$$
\mathbb{E}\left[\hat{\psi}(k)^{*} \hat{\psi}\left(k^{\prime}\right)\right]=\mathcal{E}_{0}(k) \delta\left(k-k^{\prime}\right), \quad \mathbb{E}\left[\hat{\psi}(k) \hat{\psi}\left(k^{\prime}\right)\right]=0,
$$

then the compensated wave function converges in law, as a continuous in time process taking values in an appropriate distribution space, to the solution of the time inhomogeneous stochastic equation:

$$
d \tilde{\psi}(t, k)=-\frac{\hat{\beta}(k)}{4} \tilde{\psi}(t, k) d t+\sqrt{\mathcal{R}(t, k)} d W(t, k)
$$

Here, $\mathcal{R}(t, k)$ is given by (1.13) and $\overline{\mathcal{E}}(t, k)$ is the solution of the deterministic equation (1.9) with the initial condition $\overline{\mathcal{E}}(0, k)=\mathcal{E}_{0}(k)$, while $d W(t, k)$ is a white noise on $\mathbb{R} \times \mathbb{T}$, a complex valued Gaussian process with the covariance

$$
\mathbb{E}\left[d W(t, k) d W^{*}\left(s, k^{\prime}\right)\right]=\delta\left(k-k^{\prime}\right) \otimes \delta(t-s) d t d s
$$


and $\mathcal{R}(t, k)$ is given by (1.13) . The solution of (1.16) is also explicit: $\tilde{\psi}(t)$ is the distribution

$$
\tilde{\psi}(t)=e^{-\hat{\beta} t / 4} \hat{\psi}+\int_{0}^{t} e^{-\hat{\beta}(t-s) / 4} \mathcal{R}^{1 / 2}(s) d W(s) .
$$

In particular, in the case of the initial condition distributed according to a Gibbs measure, the solution $\hat{\psi}(t, k)$ of (1.7) has the same law for all times, therefore $\overline{\mathcal{E}}(t, k)=T$ for all $t \geq 0$. In this case, (1.12) shows that $\mathcal{R}(t, k)=\hat{\beta}(k) T / 2$. Therefore, as a consequence of (1.16), the limit of the compensated wave function is the solution of the linear infinite dimensional stochastic differential equation:

$$
d \tilde{\psi}(t, k)=-\frac{\hat{\beta}(k)}{4} \tilde{\psi}(t, k) d t+\sqrt{\frac{T \hat{\beta}(k)}{2}} d W(t, k) .
$$

In the general case, when $\mathcal{E}_{0}(k)$ is not constant, we have

$$
\lim _{t \rightarrow \infty} \overline{\mathcal{E}}(t, k)=\int_{\mathbb{T}} \mathcal{E}_{0}\left(k^{\prime}\right) d k^{\prime}=T,
$$

hence, equation (1.17) describes the asymptotic stationary regime of (1.16) where the temperature is given by the average of the initial energy over all the modes $k$. Recall that the microscopic noise conserves the total energy and that the resulting temperature $T$ depends only on the law of the initial condition.

Let us also comment on the difference between the square integrable and distribution-valued initial data. While the Ornstein-Uhlenbeck equations (1.11) and (1.16) look similar, there are some important differences between them. The noises appearing in (1.11) are all of size 1 and mutually independent for different $k$-s, while the noise appearing in (1.16) is $\delta$-correlated in $k$. As a result the solution of the first equation is an ensemble of mutually independent time inhomogeneous one dimensional Ornstein-Uhlenbeck processes. On the other hand, in the case of (1.16) the resulting distribution valued Ornstein-Uhlenbeck process is $\delta$-correlated in $k$. In addition, for the square integrable data, the limit equation holds point-wise in $k$. If one considers the limit in the sense of distributions (that is, integrated against a test function) for such initial data, the stochasticity is removed, due to the fact that independent random variables, representing the solution for different modes, are simply averaged out (via the law of large numbers). As a result the limit is described simply by attenuation of the initial condition by an exponential factor $e^{-\beta(k) t / 4}$ (see part (ii) of Theorem 3.1) - that is, by (1.11) with no stochastic forcing. This result stands in sharp contrast with the case of spatially homogeneous initial data (note that then the energy has to be infinite) when the respective limit in the sense of distributions is stochastic, see (1.16), and fluctuations can not be averaged out by integration in $k$.

Finally, we note that the sole reason why we restrict ourselves to the case of one dimensional integer lattice is to avoid excessive complication of the notation that could obscure the main points of the argument. The technique of our proof can be straightforwardly applied in the case of lattice $\mathbb{Z}^{d}$. The dynamics of the corresponding perturbed system is given then by equation (45) of [4] and our results contained in Theorems 3.1 and Theorem 3.3 can be easily adjusted to deal with the case of a multidimensional lattice.

The paper is organized as follows. Section 2 contains the precise mathematical formulation of the problem and necessary definitions. We formulate the results for the convergence of 
compensated wave function in Section 3, see Theorem 3.1 for square integrable initial data, and Theorem 3.3 for spatially homogeneous, Gaussian initial distributions. The proofs of these results are presented in Sections 4 and 5, respectively.

Acknowledgement. T.K. acknowledges the support of Polish Ministry of Higher Education grant NN201419139, S.O. acknowledges the support by the ERC AdG 246953 (MALADY) and by ANR-10-BLAN 0108 (SHEPI), L.R. acknowledges the support by NSF grant DMS0908507. This work was also supported by NSSEFF fellowship by AFOSR.

\section{Preliminaries}

\subsection{Infinite system of interacting harmonic oscillators}

The dynamics of the system of oscillators can be written formally as a system of Itô stochastic differential equations indexed by $y \in \mathbb{Z}$

$$
\begin{aligned}
d \mathfrak{q}_{y}(t) & =\mathfrak{p}_{y}(t) d t \\
d \mathfrak{p}_{y}(t) & =-(\alpha * \mathfrak{q}(t))_{y} d t-\frac{\epsilon}{2}(\beta * \mathfrak{p}(t))_{y} d t+\sqrt{\epsilon} \sum_{z=-1,0,1}\left(Y_{y+z} \mathfrak{p}_{y}(t)\right) d w_{y+z}(t) .
\end{aligned}
$$

Here

$$
Y_{x}:=\left(\mathfrak{p}_{x}-\mathfrak{p}_{x+1}\right) \partial_{\mathfrak{p}_{x-1}}+\left(\mathfrak{p}_{x+1}-\mathfrak{p}_{x-1}\right) \partial_{\mathfrak{p}_{x}}+\left(\mathfrak{p}_{x-1}-\mathfrak{p}_{x}\right) \partial_{\mathfrak{p}_{x+1}}
$$

and $\left\{w_{y}(t), t \geq 0\right\}, y \in \mathbb{Z}$ is a family of i.i.d. one dimensional, real valued, standard Brownian motions, that are non-anticipative over the filtered probability space $\left(\Omega, \mathcal{F},\left\{\mathcal{F}_{t}\right\}, \mathbb{P}\right)$. In addition,

$$
\beta_{y}=\Delta \beta_{y}^{(0)}:=\beta_{y+1}^{(0)}+\beta_{y-1}^{(0)}-2 \beta_{y}^{(0)}
$$

with

$$
\beta_{y}^{(0)}=\left\{\begin{aligned}
-4, & y=0 \\
-1, & y= \pm 1 \\
0, & \text { if otherwise. }
\end{aligned}\right.
$$

Recall that the lattice Laplacian of $g: \mathbb{Z} \rightarrow \mathbb{C}$ is given by $\Delta g_{y}:=g_{y+1}+g_{y-1}-2 g_{y}$.

To understand why we choose this particular stochastic perturbation of the Hamiltonian dynamics, let us observe that we want a (continuous) noise acting only on the velocities, as local as possible, but conserving total momentum and kinetic energy. This explains why, given a site $y$, only the momenta at sites $y+z, z=-1,0,1$ are exchanged randomly. For that reason we consider the vectors $Y_{x}$ that are tangent to the local energy and momentum surfaces

$$
\mathfrak{p}_{x-1}^{2}+\mathfrak{p}_{x}^{2}+\mathfrak{p}_{x+1}^{2} \equiv \text { const }
$$

and

$$
\mathfrak{p}_{x-1}+\mathfrak{p}_{x}+\mathfrak{p}_{x+1} \equiv \text { const. }
$$

The SDE (2.1) defines a Markov process whose (formal) generator is given by

$$
L=A+\epsilon S, \quad S=\frac{1}{2} \sum_{x} Y_{x}^{2}
$$


where $A$ is the Hamiltonian vector field given by the usual Poisson brackets with the Hamiltonian. In particular $-(\beta * p)_{y} / 2=S p_{y}$.

The Fourier transform of a square integrable sequence of complex numbers $\left\{\gamma_{y}, y \in \mathbb{Z}\right\}$ is defined as

$$
\hat{\gamma}(k)=\sum_{y \in \mathbb{Z}} \gamma_{y} e_{y}(k), \quad k \in \mathbb{T} .
$$

Here

$$
e_{y}(k):=\exp \{-i 2 \pi y k\}, \quad y \in \mathbb{Z}
$$

is the standard orthonormal base in $L^{2}(\mathbb{T})$. The one dimensional torus $\mathbb{T}$ considered in this article is understood as the interval $[-1 / 2,1 / 2]$ with identified endpoints. The inverse transform is given by

$$
\check{f}_{y}=\int_{\mathbb{T}} f(k) e_{y}^{*}(k) d k, \quad y \in \mathbb{Z}
$$

for any $f$ belonging to $L^{2}(\mathbb{T})$ - the space of complex valued, square integrable functions. A simple calculation shows that

$$
\hat{\beta}(k)=8 \sin ^{2}(\pi k)\left[1+2 \cos ^{2}(\pi k)\right] .
$$

We assume also ( $\mathrm{cf}$ [4]) that

a1) $\left\{\alpha_{y}, y \in \mathbb{Z}\right\}$ is real valued and there exists $C>0$ such that $\left|\alpha_{y}\right| \leq C e^{-|y| / C}$ for all $y \in \mathbb{Z}$,

a2) $\hat{\alpha}(k)$ is also real valued and $\hat{\alpha}(k)>0$ for $k \neq 0$ and in case $\hat{\alpha}(0)=0$ we have $\hat{\alpha}^{\prime \prime}(0)>0$.

The above conditions imply that both functions $y \mapsto \alpha_{y}$ and $k \mapsto \hat{\alpha}(k)$ are even. In addition, $\hat{\alpha} \in C^{\infty}(\mathbb{T})$ and in case $\hat{\alpha}(0)=0$ we have $\hat{\alpha}(k)=\sin ^{2}(\pi k) \phi(k)$ for some strictly positive even function $\phi \in C^{\infty}(\mathbb{T})$. Recall that the function $\omega(k):=\sqrt{\hat{\alpha}(k)}$ is the dispersion relation.

\subsection{Evolution of the wave function}

For a given $m \in \mathbb{R}$ we define the space $H^{m}(\mathbb{T})$ as the completion of $C^{\infty}(\mathbb{T})$ under the norm

$$
\|f\|_{H^{m}(\mathbb{T})}^{2}:=\sum_{y \in \mathbb{Z}}\left(1+y^{2}\right)^{m}\left|\check{f}_{y}\right|^{2}
$$

We shall denote by $\langle\cdot, \cdot\rangle$ the scalar product on $L^{2}(\mathbb{T})$. By continuity it extends in an obvious way to $H^{m}(\mathbb{T}) \times H^{-m}(\mathbb{T})$ for an arbitrary $m \in \mathbb{R}$.

It is convenient to introduce the wave function that, adjusted to the macroscopic time, is given by

$$
\psi^{(\epsilon)}(t):=\check{\omega} * \mathfrak{q}\left(\frac{t}{\epsilon}\right)+i \mathfrak{p}\left(\frac{t}{\epsilon}\right)
$$

Here $\left\{\check{\omega}_{y}, y \in \mathbb{Z}\right\}$ is the inverse Fourier transform of

$$
\omega(k):=\sqrt{\hat{\alpha}(k)}
$$

We shall consider the Fourier transform of the wave function

$$
\hat{\psi}^{(\epsilon)}(t, k):=\omega(k) \hat{\mathfrak{q}}\left(\frac{t}{\epsilon}, k\right)+i \hat{\mathfrak{p}}\left(\frac{t}{\epsilon}, k\right) .
$$


Using (2.1) as a motivation, we obtain formally, by considering the Fourier transform of (2.1), that

$$
\begin{aligned}
& d \hat{\psi}^{(\epsilon)}(t)=A\left[\hat{\psi}^{(\epsilon)}(t)\right] d t+Q\left[\hat{\psi}^{(\epsilon)}(t)\right] d W(t), \\
& \hat{\psi}^{(\epsilon)}(0)=\hat{\psi}
\end{aligned}
$$

where $\hat{\psi} \in L^{2}(\mathbb{T})$, and mapping $A: L^{2}(\mathbb{T}) \rightarrow L^{2}(\mathbb{T})$ is defined by

$$
A[f](k):=-\frac{i}{\epsilon} \omega(k) f(k)-\frac{\hat{\beta}(k)}{4}\left[f_{1}(k)-f_{-1}(k)\right], \quad \forall f \in L^{2}(\mathbb{T}) .
$$

Here

$$
f_{1}(k):=f(k) \quad \text { and } \quad f_{-1}(k):=f^{*}(-k) .
$$

In addition, $Q[g]: L^{2}(\mathbb{T}) \rightarrow L^{2}(\mathbb{T})$ is a linear mapping that for any $g \in L^{2}(\mathbb{T})$ is given by

$$
Q[g](f)(k):=i \int_{\mathbb{T}} r\left(k, k^{\prime}\right)\left[g_{1}\left(k-k^{\prime}\right)-g_{-1}\left(k-k^{\prime}\right)\right] f\left(k^{\prime}\right) d k^{\prime}, \quad \forall f \in L^{2}(\mathbb{T}),
$$

where

$$
\begin{aligned}
r\left(k, k^{\prime}\right): & =\sin (2 \pi k)+\sin \left[2 \pi\left(k-k^{\prime}\right)\right]+\sin \left[2 \pi\left(k^{\prime}-2 k\right)\right] \\
& =4 \sin (\pi k) \sin \left[\pi\left(k-k^{\prime}\right)\right] \sin \left[\left(2 k-k^{\prime}\right) \pi\right], \quad k, k^{\prime} \in \mathbb{T} .
\end{aligned}
$$

The cylindrical Wiener process on $L^{2}(\mathbb{T})$ appearing in (2.12) is $d W(t):=\sum_{y \in \mathbb{Z}} e_{y} d w_{y}(t)$.

It can be easily checked that $\sum_{y \in \mathbb{Z}}\left\|Q[g]\left(e_{y}\right)\right\|_{L^{2}(\mathbb{T})}^{2} \leq C\|g\|_{L^{2}(\mathbb{T})}^{2}$ for some $C>0$ and all $g \in L^{2}(\mathbb{T})$ so $Q[g]$ is Hilbert-Schmidt, which ensures that

$$
Q\left[\hat{\psi}^{(\epsilon)}(t)\right] d W(t):=\sum_{y \in \mathbb{Z}} Q\left[\hat{\psi}^{(\epsilon)}(t)\right]\left(e_{y}\right) d w_{y}(t)
$$

is summable in $L^{2}(\mathbb{T})$, both in the $L^{2}$ and a.s. sense. It is also obvious that the mapping $A$ is Lipschitz. Using Theorem 7.4, p. 186, of [7] one concludes therefore that there exists an $L^{2}(\mathbb{T})$-valued, adapted process $\left\{\hat{\psi}^{(\epsilon)}(t), t \geq 0\right\}$ that is a unique solution to (2.12). In addition, see Section 2 of [4], the total energy is conserved:

$$
\left\|\hat{\psi}^{(\epsilon)}(t)\right\|_{L^{2}(\mathbb{T})}=\text { const, } \quad \forall t \geq 0
$$

for a.s. realization of Brownian motions and an initial condition from $L^{2}(\mathbb{T})$.

\subsection{Compensated wave function}

Let us define the compensated wave function

$$
\tilde{\psi}^{(\epsilon)}(t, k):=\hat{\psi}^{(\epsilon)}(t, k) \exp \left\{i t \frac{\omega(k)}{\epsilon}\right\} .
$$

From (2.12) we obtain the following equation

$$
\begin{aligned}
& d \tilde{\psi}^{(\epsilon)}(t, k)=\mathcal{A}\left[\frac{t}{\epsilon}, \tilde{\psi}^{(\epsilon)}(t)\right](k) d t+d \tilde{\mathcal{M}}_{t}^{(\epsilon)}(k), \\
& \tilde{\psi}^{(\epsilon)}(0)=\hat{\psi},
\end{aligned}
$$


where $\hat{\psi} \in L^{2}(\mathbb{T}), \mathcal{A}[t, \cdot]: L^{2}(\mathbb{T}) \rightarrow L^{2}(\mathbb{T})$

$$
\mathcal{A}[t, f](k):=-\frac{\hat{\beta}(k)}{4}\left[f(k)-\exp \{2 i \omega(k) t\} f^{*}(-k)\right] .
$$

The martingale term equals

$$
d \tilde{\mathcal{M}}_{t}^{(\epsilon)}:=\tilde{Q}\left[\frac{t}{\epsilon}, \tilde{\psi}^{(\epsilon)}(t)\right] d W(t)
$$

where for any $g \in L^{2}(\mathbb{T})$ and $t \geq 0$, the operator $\tilde{Q}[t, g]: L^{2}(\mathbb{T}) \rightarrow L^{2}(\mathbb{T})$, is given by

$$
\tilde{Q}[t, g](f)(k):=i \sum_{\sigma= \pm 1} \sigma \int_{\mathbb{T}} r\left(k, k^{\prime}\right) g_{\sigma}\left(k-k^{\prime}\right) f\left(k^{\prime}\right) \exp \left\{i\left[\omega(k)-\sigma \omega\left(k-k^{\prime}\right)\right] t\right\} d k^{\prime} .
$$

Using a standard theory of S.P.D.E.-s, see [7], we can show the following result.

Proposition 2.1 Suppose that $-3 / 2<m<1$. If the initial condition $\hat{\psi}(\cdot)$ belongs to $H^{m}(\mathbb{T})$ then there exists a unique solution $\left(\tilde{\psi}^{(\epsilon)}(t)\right)$ of (2.17) in $H^{m}(\mathbb{T})$.

The proof of this result shall be presented in Appendix A. Since the dispersion relation $\omega(\cdot)$ might not be differentiable in the classical sense at 0 (but it belongs to $H^{1}(\mathbb{T})$ ) we cannot guarantee better regularity of the solutions of (2.17). Recall that the classical Sobolev embedding theorem ensures that $H^{m}(\mathbb{T})$, for $m>1 / 2$, is embedded in the space of continuous functions on the torus $C(\mathbb{T})$, see e.g. Theorem 7.10, p. 155 of $[8]$.

\section{Convergence of the compensated process}

\subsection{Square integrable initial data}

Before formulating the result we introduce some auxiliaries. First, for any $k_{1}, k_{2} \in \mathbb{T}$ let us denote

$$
\mathcal{K}\left(k_{1}, k_{2}\right)=\bigcup_{\sigma_{1}, \sigma_{2}, \sigma_{3}= \pm 1}\left[k: \omega\left(k_{1}\right)+\sigma_{3} \omega\left(k-k_{1}\right)=\sigma_{1}\left[\omega\left(k_{2}\right)+\sigma_{2} \omega\left(k-k_{2}\right)\right]\right]
$$

We shall require that:

Condition $\omega$ ) for any $k_{1} \neq k_{2}$ the one dimensional Lebesgue measure $m_{1}\left(\mathcal{K}\left(k_{1}, k_{2}\right)\right)=0$.

More detailed discussion of this condition shall be carried out in Remark 2 after Theorem 3.1 below.

Define the scattering operator $\mathcal{L}: L^{1}(\mathbb{T}) \rightarrow L^{1}(\mathbb{T})$ by

$$
\mathcal{L} f(k):=\int_{\mathbb{T}} R\left(k, k^{\prime}\right)\left[f\left(k^{\prime}\right)-f(k)\right] d k^{\prime}, \quad f \in L^{1}(\mathbb{T}),
$$

where the scattering kernel is given by

$$
\begin{aligned}
& R\left(k, k^{\prime}\right):=r^{2}\left(k, k-k^{\prime}\right)+r^{2}\left(k, k+k^{\prime}\right) \\
& \quad=16 \sin ^{2}(\pi k) \sin ^{2}\left(\pi k^{\prime}\right)\left\{\sin ^{2}\left[\pi\left(k+k^{\prime}\right)\right]+\sin ^{2}\left[\pi\left(k-k^{\prime}\right)\right]\right\} .
\end{aligned}
$$


Suppose that $\hat{\psi} \in L^{2}(\mathbb{T})$. Let

$$
\mathcal{R}(t, k):=\int_{\mathbb{T}} R\left(k, k^{\prime}\right) \overline{\mathcal{E}}\left(t, k^{\prime}\right) d k^{\prime}
$$

where $\overline{\mathcal{E}}(t, k)$ is the unique solution in $C\left(\mathbb{R}, L^{1}(\mathbb{T})\right)$ of an equation

$$
\overline{\mathcal{E}}(t, k)=|\hat{\psi}(k)|^{2}+\int_{0}^{t} \mathcal{L} \overline{\mathcal{E}}(s, k) d s .
$$

The existence and uniqueness of solutions in (3.4) follows from the fact that $\mathcal{L}$ is clearly a bounded operator on $L^{1}(\mathbb{T})$. The solution then is given by $\overline{\mathcal{E}}(t)=P^{t} \overline{\mathcal{E}}(0)$, where $\overline{\mathcal{E}}(0):=|\hat{\psi}|^{2}$ and $\left(P^{t}\right)$ is the contraction semigroup on $L^{1}(\mathbb{T})$ generated by $\mathcal{L}$.

Assume also that $\left\{w_{k}(t), t \geq 0\right\}$ is a family of pairwise independent standard, one dimensional, complex valued Brownian motions indexed by $k \in \mathbb{T}$. Our first principal result can be stated as follows.

Theorem 3.1 Suppose that the dispersion relation $\omega(\cdot)$ satisfies condition $\omega)$. Then, the following are true:

(i) if $\hat{\psi} \in H^{m}(\mathbb{T})$ for some $m>1 / 2$ then there exists a solution $\tilde{\psi}^{(\epsilon)}(t)$ of (2.17) that belongs a.s. to $C(\mathbb{T})$ for all $t \geq 0$. In addition, given an integer $n \geq 1$ and $k_{1}, \ldots, k_{n} \in \mathbb{T}$, the processes $\left\{\left(\tilde{\psi}^{(\epsilon)}\left(t, k_{1}\right), \ldots, \tilde{\psi}^{(\epsilon)}\left(t, k_{n}\right)\right), t \geq 0\right\}$ converge in law over $C\left([0,+\infty) ; \mathbb{C}^{n}\right)$, as $\epsilon \rightarrow 0+$, to $\left\{\left(\tilde{\psi}\left(t, k_{1}\right), \ldots, \tilde{\psi}\left(t, k_{n}\right)\right), t \geq 0\right\}$, where $\{\tilde{\psi}(t, k), t \geq 0\}$ is a complex valued, non-homogeneous in time Ornstein-Uhlenbeck process that is the solution of the equation

$$
\begin{aligned}
d \tilde{\psi}(t, k) & =-\frac{\hat{\beta}(k)}{4} \tilde{\psi}(t, k) d t+\mathcal{R}^{1 / 2}(t, k) d w_{k}(t), \\
\tilde{\psi}(0, k) & =\hat{\psi}(k),
\end{aligned}
$$

(ii) if $\hat{\psi} \in L^{2}(\mathbb{T})$, then for any $f \in L^{2}(\mathbb{T})$ and $t_{*}>0$ we have

$$
\lim _{\epsilon \rightarrow 0+} \sup _{t \in\left[0, t_{*}\right]}\left|\left\langle\tilde{\psi}^{(\epsilon)}(t)-\bar{\psi}(t), f\right\rangle\right|=0
$$

in probability. Here $\bar{\psi}(t)$ is given by

$$
\bar{\psi}(t, k):=\hat{\psi}_{0}(k) \exp \left\{-\frac{t \hat{\beta}(k)}{4}\right\} .
$$

Remark 1. We claim that

$$
\lim _{t \rightarrow+\infty} \sup _{k \in \mathbb{T}}|\mathcal{R}(t, k)-(\hat{\beta}(k) / 2) T|=0
$$

where $T=\left\|\hat{\psi}_{0}\right\|_{L^{2}(\mathbb{T})}^{2}$. The above easily follows from (3.3), provided we show that any solution $\overline{\mathcal{E}}(t, k)$ of (3.4) satisfies

$$
\lim _{t \rightarrow+\infty}\|\overline{\mathcal{E}}(t)-T\|_{L^{1}(\mathbb{T})}=0 .
$$

To prove (3.9) recall that operator $\mathcal{L}$ given by $(3.4)$ is a generator of a strongly continuous semigroup $\left(P^{t}\right)$ of contractions on $L^{1}(\mathbb{T})$. In fact, it is also a semigroup of contractions when 
restricted to any $L^{p}(\mathbb{T})$, for $1 \leq p \leq+\infty$, strongly continuous, provided that $p \in[1,+\infty)$. When $p=2$ generator $\mathcal{L}$ is symmetric (and so is each $P^{t}$ ) and

$$
\langle\mathcal{L} f, f\rangle=-\frac{1}{2} \int_{\mathbb{T}^{2}} R\left(k, k^{\prime}\right)\left|f\left(k^{\prime}\right)-f(k)\right|^{2} d k d k^{\prime} \leq 0, \quad \forall f \in L^{2}(\mathbb{T}) .
$$

Hence 0 is a simple eigenvalue of $\mathcal{L}$ in $L^{2}(\mathbb{T})$, i.e. if $f \in L^{2}(\mathbb{T})$ and satisfies $\mathcal{L} f=0$, then $f$ is a constant. This immediately implies that for $\overline{\mathcal{E}}(0) \in L^{2}(\mathbb{T})$ with $T:=\int_{\mathbb{T}} \overline{\mathcal{E}}(0, k) d k$ we have

$$
\lim _{t \rightarrow+\infty}\|\overline{\mathcal{E}}(t)-T\|_{L^{2}(\mathbb{T})}^{2}=\lim _{t \rightarrow+\infty} \int_{0}^{+\infty} e^{-\lambda t} \mu(d \lambda)=0,
$$

where $\mu$ is the spectral measure of $\overline{\mathcal{E}}(0)-T$ corresponding to $\mathcal{L}$. This in particular implies (3.9) in case the initial data is square integrable. If $\overline{\mathcal{E}}(0)$ only belongs to $L^{1}(\mathbb{T})$ we obtain (3.9) approximating first $\overline{\mathcal{E}}(0)$ by square integrable functions and then using (3.10) together with the fact that $\left(P^{t}\right)$ is a contraction semigroup on $L^{1}(\mathbb{T})$.

From (3.8) we obtain, for any $k \in \mathbb{T}$,

$$
\lim _{t \rightarrow+\infty} \mathbb{E}\left|\tilde{\psi}(t, k)-\tilde{\psi}_{s}(t, k)\right|^{2}=0
$$

where $\tilde{\psi}_{s}(t, k)$ is a time homogeneous Ornstein-Uhlenbeck process given by

$$
\begin{aligned}
d \tilde{\psi}_{s}(t, k) & =-\frac{\hat{\beta}(k)}{4} \tilde{\psi}_{s}(t, k) d t+\sqrt{\frac{\hat{\beta}(k) T}{2}} d w_{k}(t), \\
\tilde{\psi}_{s}(0, k) & =\hat{\psi}(k) .
\end{aligned}
$$

Remark 2. Let us also comment briefly on condition $\omega$ ). A similar hypothesis appears in the wave turbulence theory under the name of a no resonance condition, see e.g. [20]. In our context we use it, among others, to prove the asymptotic (in the limit $\epsilon \rightarrow 0+$ ) independence of $\tilde{\psi}^{(\epsilon)}(t, k)$ for different $k$. This independence implies, in particular, the self-averaging property of the energy $\left|\tilde{\psi}^{(\epsilon)}(t, k)\right|^{2}$ i.e. its convergence in probability to a deterministic limit, as $\epsilon \rightarrow 0+$, in the weak topology, see Proposition 4.1 below. This observation plays a crucial rôle in the proof of part (i) of Theorem 3.1. Without lack of resonance condition of the type $\omega$ ), it is in principle possible that the second mixed moment of the energy corresponding to different modes does not vanish in the limit, as $\epsilon \rightarrow 0+$, so that the key estimate (4.26) below fails making self-averaging of energy impossible.

The following simple criterion is useful for verification of condition $\omega)$, e.g. for dispersion relation $\omega(k)$ of the form (1.4). Recall that from the assumptions made we know that $\omega \in$ $C^{\infty}(\mathbb{T} \backslash\{0\})$.

Lemma 3.2 Suppose that the dispersion relation $\omega(\cdot)$ satisfies the following condition: for any $|a|<1 / 2$ and $\sigma= \pm 1$ the set of solutions of an equation

$$
\omega^{\prime}(k)=\sigma \omega^{\prime}(k+a)
$$

is possibly of positive Lebesgue measure in $\mathbb{T}$, only if $a=0$ and $\sigma=1$. Then, for any $\left(k_{1}, k_{2}\right)$ such that $k_{1} \neq k_{2}$ the hypothesis $\omega$ ) holds. 
Proof. Fix $\left(k_{1}, k_{2}\right)$ such that $k_{1} \neq k_{2}$. To simplify we consider only the set $\mathcal{K}_{1}$ that corresponds to $\sigma_{1}=\sigma_{2}=\sigma_{3}=1$ and prove that:

$$
\mathcal{K}_{1}\left(k_{1}, k_{2}\right):=\left[k: \omega\left(k_{1}\right)+\omega\left(k-k_{2}\right)=\omega\left(k_{2}\right)+\omega\left(k-k_{1}\right)\right]
$$

is of null Lebesgue measure. The remaining cases can be dealt with similarly. Suppose, on the contrary, that the Lebesgue measure of the set is positive. Then almost every point of $\mathcal{K}_{1}\left(k_{1}, k_{2}\right)$ is a density point of the set. In particular that means that at any such point we have

$$
\omega^{\prime}\left(k-k_{2}\right)=\omega^{\prime}\left(k-k_{1}\right)
$$

but this would clearly contradict the assumption made in the statement of the lemma.

It is quite straightforward to verify that the above lemma applies to the dispersion relation of the form (1.4).

\subsection{Statistically homogeneous initial data}

For a given non-negative $m$ we assume that the initial data $\hat{\psi}$ is an $H^{-m}(\mathbb{T})$ valued Gaussian random element. More precisely, suppose that $\mathcal{E}_{0}(\cdot)$ is a non-negative function such that

$$
\sum_{x \in \mathbb{Z}}\left|\left\langle\mathcal{E}_{0}, e_{x}\right\rangle\right|<+\infty
$$

$\left\{\xi_{y}, y \in \mathbb{Z}\right\}$ are i.i.d. complex Gaussian random variables such that $\mathbb{E} \xi_{0}=0$ and $\mathbb{E}\left|\xi_{0}\right|^{2}=1$, and

$$
\hat{\psi}(k)=\sum_{y \in \mathbb{Z}} \xi_{y} \mathcal{E}_{0}^{1 / 2}(k) e_{y}(k) .
$$

The law of $\hat{\psi}$ is supported in $H^{-m}(\mathbb{T})$, provided that $m>1 / 2$. Its covariance form equals

$$
\mathcal{C}\left(J_{1}, J_{2}\right):=\mathbb{E}\left[\left\langle J_{1}, \hat{\psi}\right\rangle\left\langle J_{2}, \hat{\psi}\right\rangle^{*}\right]=\int_{\mathbb{T}} \mathcal{E}_{0}(k) J_{1}(k) J_{2}^{*}(k) d k
$$

for any $J_{1}, J_{2} \in C^{\infty}(\mathbb{T})$. The Gibbs equilibrium states described in the introduction correspond to $\mathcal{E}_{0}(k) \equiv$ const. Using Proposition 2.1 we conclude that equation (2.17) has a unique mild solution $\left\{\tilde{\psi}^{(\epsilon)}(t), t \geq 0\right\}$ whose realizations belong to $C\left([0,+\infty) ; H^{-m}(\mathbb{T})\right)$, provided $m<3 / 2$.

Let $\mathcal{R}(t, k)$ be given by (3.3) with $\overline{\mathcal{E}}(t, k)$ the solution of (3.4) satisfying $\overline{\mathcal{E}}(0, k)=\mathcal{E}_{0}(k)$. Observe that the operator $f(k) \mapsto \mathcal{R}^{1 / 2}(t, k) f(k)$ is Hilbert-Schmidt, when considered from $L^{2}(\mathbb{T})$ to $H^{-m}(\mathbb{T})$, provided $m>1 / 2$. Indeed

$$
\sum_{y}\left\|\mathcal{R}^{1 / 2}(t) e_{y}\right\|_{H^{-m}(\mathbb{T})}^{2}=\sum_{y, y_{1}}\left(1+y_{1}^{2}\right)^{-m}\left|\int \mathcal{R}^{1 / 2}(t, k) e_{y-y_{1}}(k) d k\right|^{2} .
$$

By Plancherel's identity the right hand side equals

$$
\begin{aligned}
& \sum_{y_{1}}\left(1+y_{1}^{2}\right)^{-m} \sum_{z}\left|\int \mathcal{R}^{1 / 2}(t, k) e_{z}(k) d k\right|^{2}=\sum_{y_{1}}\left(1+y_{1}^{2}\right)^{-m}\left\|\mathcal{R}^{1 / 2}(t, \cdot)\right\|_{L^{2}(\mathbb{T})}^{2} \\
& =\sum_{y_{1}}\left(1+y_{1}^{2}\right)^{-m}\|\mathcal{R}(t, \cdot)\|_{L^{1}(\mathbb{T})}^{2}<+\infty
\end{aligned}
$$


Since in addition $f(k) \mapsto-(\hat{\beta}(k) / 4) f(k)$ is bounded on $H^{-m}(\mathbb{T})$, the equation

$$
\begin{aligned}
d \bar{\psi}_{*}(t, k) & =-\frac{\hat{\beta}(k)}{4} \bar{\psi}_{*}(t, k) d t+\mathcal{R}^{1 / 2}(t, k) d W(t, k), \\
\bar{\psi}_{*}(0, k) & =\hat{\psi}(k)
\end{aligned}
$$

has a unique $H^{-m}(\mathbb{T})$-valued mild solution, by virtue of Theorem 7.4, p. 186 of [7]. It is given by the formula

$$
\bar{\psi}_{*}(t, k)=e^{-\hat{\beta}(k) t / 4} \hat{\psi}+\int_{0}^{t} e^{-\hat{\beta}(k)(t-s) / 4} \mathcal{R}^{1 / 2}(s, k) d W(s, k) .
$$

We denote by $H_{w}^{-m}(\mathbb{T})$ the Hilbert space equipped with the weak topology. Our main result is as follows.

Theorem 3.3 Suppose that $3 / 2>m>1 / 2$ and both (3.14) and condition $\omega)$ hold. Then, under the above assumptions, the processes $\left\{\tilde{\psi}^{(\epsilon)}(t), t \geq 0\right\}$ converge in law over $C\left([0,+\infty), H_{w}^{-m}(\mathbb{T})\right)$, as $\epsilon \rightarrow 0+$, to $\left\{\bar{\psi}_{*}(t), t \geq 0\right\}$.

Remark. As in the remark made after Theorem 3.1 we can also conclude that

$$
\lim _{t \rightarrow+\infty} \mathbb{E}\left|\left\langle\bar{\psi}_{*}(t)-\bar{\psi}_{s}(t), f\right\rangle\right|^{2}=0,
$$

where $\bar{\psi}_{s}(t)$ is a time homogeneous, distribution valued Ornstein-Uhlenbeck process given by

$$
\begin{aligned}
d \bar{\psi}_{s}(t, k) & =-\frac{\hat{\beta}(k)}{4} \bar{\psi}_{s}(t, k) d t+\sqrt{\frac{\hat{\beta}(k) T}{2}} d W(t, k), \\
\bar{\psi}_{s}(0, k) & =\hat{\psi}(k),
\end{aligned}
$$

where $T=\left\|\mathcal{E}_{0}\right\|_{L^{1}(\mathbb{T})}$.

\section{Proof of Theorem 3.1}

The fact that the solution of (2.17) lies in $C(\mathbb{T})$ for each $\epsilon>0$ is a direct consequence of Proposition 2.1 and the embedding of $H^{m}(\mathbb{T})$ into $C(\mathbb{T})$ for $m>1 / 2$. We prove first the part $(i)$ of the theorem. To explain the idea of the proof assume that $n=1$ (that is, the process $\hat{\psi}(t, k)$ for a fixed $k$ ), the independence of the compensated wave function for various $k$ is handled in the same manner. Since the coefficients appearing in the stochastic differential equation describing the evolution of $\tilde{\psi}^{(\epsilon)}(t)$ (see (2.17) ) are of the order $O(1)$, it is easy to conclude that for each $k$ the laws of the processes $\left\{\tilde{\psi}^{(\epsilon)}(t, k), t \geq 0\right\}$ are tight over $C([0,+\infty) ; \mathbb{C})$, as $\epsilon \rightarrow 0+$. In order to identify the limit, thus proving part i) of the theorem, we have to deal with the rapidly oscillating terms. First, we show that the rapidly oscillating part of the bounded variation term in (2.17) (with the factor $\exp \{2 i \omega(k) t / \epsilon\}$ in (2.18)) vanishes in the limit thanks to part i) of Corollary 4.3 below.

Next, the limit of the martingale part $\tilde{\mathcal{M}}_{t}^{(\epsilon)}(k)$ in (2.17) is a complex Gaussian martingale with the quadratic variation equal to $\int_{0}^{t} \mathcal{R}(s, k) d s$ thanks to the following:

$$
\lim _{\epsilon \rightarrow 0+} \sup _{t \in\left[0, t_{*}\right]}\left|\left\langle\tilde{\mathcal{M}}^{(\epsilon)}(k),\left(\tilde{\mathcal{M}}^{(\epsilon)}\right)^{*}(k)\right\rangle_{t}-\int_{0}^{t} \mathcal{R}(s, k) d s\right|=0,
$$


where the convergence holds in probability, for any $t_{*}>0$. This is done in Proposition 4.1, The method of proof of (4.1) is as follows. From (2.19), we compute the quadratic variation:

$$
\begin{aligned}
& \left\langle\tilde{\mathcal{M}}^{(\epsilon)}(k),\left(\tilde{\mathcal{M}}^{(\epsilon)}\right)^{*}(k)\right\rangle_{t} \\
& =\sum_{\sigma_{1}, \sigma_{2}= \pm 1} \sigma_{1} \sigma_{2} \int_{0}^{t} d s \int_{\mathbb{T}} r^{2}\left(k, k^{\prime}\right) \hat{\psi}_{\sigma_{1}}^{(\epsilon)}\left(s, k-k^{\prime}\right)\left(\hat{\psi}_{\sigma_{2}}^{(\epsilon)}\right)^{*}\left(s, k-k^{\prime}\right) d k^{\prime} .
\end{aligned}
$$

The terms appearing in (4.2) are of the following form:

$$
\begin{aligned}
& \mathcal{V}_{\epsilon}^{(0)}(t):=\int_{0}^{t}\left\langle\left|\hat{\psi}^{(\epsilon)}(s)\right|^{2}, f\right\rangle d s, \\
& \mathcal{V}_{\epsilon}^{(1)}(t):=\int_{0}^{t} \int_{\mathbb{T}} \hat{\psi}^{(\epsilon)}(s, k) \hat{\psi}^{(\epsilon)}(s,-k) f^{*}(k) d k d s .
\end{aligned}
$$

Here $f(k)$ is a certain explicit function related to the scattering kernel. As $\hat{\psi}^{(\epsilon)}(t, k)$ (without the compensation) is rapidly oscillating as $e^{-i \omega(k) t / \epsilon}$, therefore we expect that only $\mathcal{V}_{\epsilon}^{(0)}(t)$ has a non-trivial limit. This term contains no oscillation and is essentially the time integral of scattered energy $\left|\hat{\psi}^{(\epsilon)}(t, k)\right|^{2}$. It has been shown in [4] that the expectation of the energy converges to the solution of (1.9). We need to strengthen this result to convergence in probability.

The proof of part ii) of the theorem uses the same ideas. Integrating against a test function results in the formula for the quadratic variation, see (4.34), containing only terms with fast oscillating factors, so the stochastic part vanishes in the limit.

We now turn to the proof of part (i) the theorem. In particular, we assume that $\hat{\psi} \in H^{m}$, $m>1 / 2$ so that $\tilde{\psi}^{(\epsilon)}(t, k)$ is continuous and point-wise evaluations in $k$ make sense. An application of the Itô formula to (2.12) yields, see Theorem 4.17 of [7],

$$
d\left|\hat{\psi}^{(\epsilon)}(t, k)\right|^{2}=\left[I_{\epsilon}(t, k)+I I_{\epsilon}(t, k)\right] d t+d \mathcal{M}_{t}^{(\epsilon)}(k)+d \mathcal{M}_{t}^{(\epsilon) *}(k),
$$

where

$$
\begin{aligned}
& I_{\epsilon}(t, k):=\left(A\left[\hat{\psi}^{(\epsilon)}(t)\right]\right)^{*}(k) \hat{\psi}^{(\epsilon)}(t, k)+\left(\hat{\psi}^{(\epsilon)}\right)^{*}(t, k) A\left[\hat{\psi}^{(\epsilon)}(t)\right](k), \\
& I_{\epsilon}(t, k):=\sum_{y \in \mathbb{Z}}\left|Q\left[\hat{\psi}^{(\epsilon)}(t)\right]\left(e_{y}\right)(k)\right|^{2},
\end{aligned}
$$

and $\mathcal{M}_{t}^{(\epsilon)}$ is an $\mathcal{F}_{t}$-adapted local martingale, given by

$$
\mathcal{M}_{t}^{(\epsilon)}(k)=\int_{0}^{t} \hat{\psi}^{(\epsilon)}(s, k)\left(Q\left[\hat{\psi}^{(\epsilon)}(s)\right] d W(s)\right)^{*}(k) .
$$

From (2.13) we obtain that

$$
I_{\epsilon}(t, k)=-\frac{\hat{\beta}(k)}{2}\left|\hat{\psi}^{(\epsilon)}(t, k)\right|^{2}-\frac{\hat{\beta}(k)}{4} \hat{\psi}_{2}^{(\epsilon)}(t, k),
$$

where

$$
\hat{\psi}_{2}^{(\epsilon)}(t, k):=\hat{\psi}^{(\epsilon)}(t, k) \hat{\psi}^{(\epsilon)}(t,-k)+\left(\hat{\psi}^{(\epsilon)}\right)^{*}(t, k)\left(\hat{\psi}^{(\epsilon)}\right)^{*}(t,-k),
$$


while equation (2.15) yields

$$
I I_{\epsilon}(t, k)=\int_{\mathbb{T}} R\left(k, k^{\prime}\right)\left|\hat{\psi}^{(\epsilon)}\left(t, k^{\prime}\right)\right|^{2} d k^{\prime}+\frac{1}{2} \int_{\mathbb{T}} R\left(k, k^{\prime}\right) \hat{\psi}_{2}^{(\epsilon)}\left(t, k^{\prime}\right) d k^{\prime} .
$$

Analogous equation can be derived for $d\left[\hat{\psi}^{(\epsilon)}(t, k) \hat{\psi}^{(\epsilon)}(t,-k)\right]$. The corresponding terms shall be denoted by $\tilde{I}_{\epsilon}(t, k), \tilde{I I}_{\epsilon}(t, k)$ and the martingale $\mathcal{N}_{t}^{(\epsilon, 1)}(k)+\mathcal{N}_{t}^{(\epsilon, 2)}(k)$, where

$$
\begin{gathered}
\tilde{I}_{\epsilon}(t, k)=-\frac{2 i \omega(k)}{\epsilon} \hat{\psi}_{2}^{(\epsilon)}(t, k)+\mathcal{P}\left[\hat{\psi}^{(\epsilon)}(t),\left(\hat{\psi}^{(\epsilon)}\right)^{*}(t)\right], \\
I I_{\epsilon}(t, k)=\mathcal{Q}\left[\hat{\psi}^{(\epsilon)}(t),\left(\hat{\psi}^{(\epsilon)}\right)^{*}(t)\right],
\end{gathered}
$$

where $\mathcal{P}, \mathcal{Q}$ are second degree polynomials in $\hat{\psi}^{(\epsilon)}(t),\left(\hat{\psi}^{(\epsilon)}\right)^{*}(t)$, and

$$
\begin{aligned}
& \mathcal{N}_{t}^{(\epsilon, 1)}(k)=\int_{0}^{t} \hat{\psi}^{(\epsilon)}(s,-k)\left(Q\left[\hat{\psi}^{(\epsilon)}(s)\right] d W(s)\right)(k), \\
& \mathcal{N}_{t}^{(\epsilon, 2)}(k)=\int_{0}^{t} \hat{\psi}^{(\epsilon)}(s, k)\left(Q\left[\left(\hat{\psi}_{-1}^{(\epsilon)}\right)^{*}(s)\right] d W(s)\right)(-k) .
\end{aligned}
$$

Proposition 4.1 Let $f \in L^{\infty}(\mathbb{T}), \mathcal{V}_{\epsilon}^{(0)}(t)$ given by (4.3), and let $\mathcal{V}_{\epsilon, a}^{(1)}(t)$ be defined by

$$
\mathcal{V}_{\epsilon, a}^{(1)}(t):=\int_{0}^{t} \int_{\mathbb{T}} \exp \left\{\frac{i s a}{\epsilon}\right\} \hat{\psi}^{(\epsilon)}(s, k) \hat{\psi}^{(\epsilon)}(s,-k) f^{*}(k) d k d s, \quad a \in \mathbb{R} .
$$

Then, for any $t_{*}>0$ we have

$$
\lim _{\epsilon \rightarrow 0+} \sup _{t \in\left[0, t_{*}\right]}\left|\mathcal{V}_{\epsilon}^{(0)}(t)-\int_{0}^{t}\langle\overline{\mathcal{E}}(s), f\rangle d s\right|=0
$$

and

$$
\lim _{\epsilon \rightarrow 0+} \sup _{t \in\left[0, t_{*}\right]}\left|\mathcal{V}_{\epsilon, a}^{(1)}(t)\right|=0, \quad a \in \mathbb{R}
$$

in probability.

The proof of this proposition shall be obtained at the end of a series of lemmas.

Lemma 4.2 For any $p \in[2,+\infty)$ there exists $C>0$ such that, for any $t_{*}>0$

$$
\sup _{\epsilon \in(0,1]} \mathbb{E}\left[\sup _{t \in\left[0, t_{*}\right]}\left\|\hat{\psi}^{(\epsilon)}(t)\right\|_{L^{p}(\mathbb{T})}^{p}\right] \leq C e^{C t_{*}}\|\hat{\psi}\|_{L^{p}(\mathbb{T})}^{p},
$$

and,

$$
\sup _{\epsilon \in(0,1], k \in \mathbb{T}} \mathbb{E}\left[\sup _{t \in\left[0, t_{*}\right]}\left|\hat{\psi}^{(\epsilon)}(t, k)\right|^{p}\right] \leq C e^{C t_{*}}\|\hat{\psi}\|_{L^{p}(\mathbb{T})}^{p}
$$


Proof. Let

$$
T_{t}^{(\epsilon)} \hat{\psi}(k):=\exp \left\{-i \frac{\omega(k) t}{\epsilon}\right\} \hat{\psi}(k), \quad \hat{\psi} \in L^{p}(\mathbb{T}), t \in \mathbb{R} .
$$

We obviously have

$$
\left\|T_{t}^{(\epsilon)} \hat{\psi}\right\|_{L^{p}(\mathbb{T})}=\|\hat{\psi}\|_{L^{p}(\mathbb{T})}, \quad \forall t \geq 0 .
$$

Using the Duhamel formula, the solution of (2.12) can be written as

$$
\hat{\psi}^{(\epsilon)}(t, k)=\hat{\psi}(k)+\int_{0}^{t} T_{t-s}^{(\epsilon)} B\left[\hat{\psi}^{(\epsilon)}(s)\right](k) d s+\int_{0}^{t} T_{t-s}^{(\epsilon)} Q\left[\hat{\psi}^{(\epsilon)}(s)\right] d W(s, k),
$$

where $B f(k)=-\hat{\beta}(k)\left[f(k)-f^{*}(-k)\right] / 4$. Hence, for a given $\epsilon \in(0,1]$ and $t_{0}>0$ to be adjusted later on, we can write

$$
\begin{aligned}
& \mathbb{E}\left[\sup _{t \in\left[0, t_{0}\right]}\left|\hat{\psi}^{(\epsilon)}(t, k)\right|^{p}\right] \leq C\left\{|\hat{\psi}(k)|^{p}+t_{0}^{p-1} \int_{0}^{t_{0}} \mathbb{E}\left|\hat{\psi}^{(\epsilon)}(s, k)\right|^{p} d s\right. \\
& \left.+\mathbb{E}\left\{\sup _{t \in\left[0, t_{0}\right]}\left|\int_{0}^{t} T_{-s}^{(\epsilon)} Q\left[\hat{\psi}^{(\epsilon)}(s)\right] d W(s, k)\right|^{p}\right\}\right\} .
\end{aligned}
$$

To estimate the martingale term on the right hand side we use Burkholder-Davis-Gundy inequality which allows to bound it by

$$
4^{p / 2} \mathbb{E}\left(\int_{0}^{t_{0}} \int_{\mathbb{T}} R\left(k, k^{\prime}\right)\left|\hat{\psi}^{(\epsilon)}\left(s, k-k^{\prime}\right)\right|^{2} d k^{\prime} d s\right)^{p / 2} \leq C_{1} t_{0}^{p / 2-1} \int_{0}^{t_{0}} \mathbb{E}\left\|\hat{\psi}^{(\epsilon)}(s)\right\|_{L^{p}(\mathbb{T})}^{p} d s
$$

for some constant $C_{1}>0$. Choosing $t_{0}$ sufficiently small, so that $C t_{0}^{p}+C C_{1} t_{0}^{p / 2}<1 / 2$, we conclude that

$$
\mathbb{E}\left\{\sup _{t \in\left[0, t_{0}\right]}\left\|\hat{\psi}^{(\epsilon)}(t)\right\|_{L^{p}(\mathbb{T})}^{p}\right\} \leq 2 C\|\hat{\psi}\|_{L^{p}(\mathbb{T})}^{p} .
$$

The argument leading to (4.15) can be used on each of the intervals $\left[j t_{0},(j+1) t_{0}\right)$ for any $j \geq 1$ and yields

$$
\mathbb{E}\left\{\sup _{t \in\left[j t_{0},(j+1) t_{0}\right]}\left\|\hat{\psi}^{(\epsilon)}(t)\right\|_{L^{p}(\mathbb{T})}^{p}\right\} \leq C \mathbb{E}\left\|\hat{\psi}^{(\epsilon)}\left(j t_{0}\right)\right\|_{L^{p}(\mathbb{T})}^{p} \leq C \mathbb{E}\left\{\sup _{t \in\left[(j-1) t_{0}, j t_{0}\right]}\left\|\hat{\psi}^{(\epsilon)}(t)\right\|_{L^{p}(\mathbb{T})}^{p}\right\},
$$

for some constant $C>0$ independent of $j$ and $\epsilon \in(0,1]$. Hence, after $j$ iterations of the above estimate, we conclude

$$
\mathbb{E}\left\{\sup _{t \in\left[j t_{0},(j+1) t_{0}\right]}\left\|\hat{\psi}^{(\epsilon)}(t)\right\|_{L^{p}(\mathbb{T})}^{p}\right\} \leq C^{j}\|\hat{\psi}\|_{L^{p}(\mathbb{T})}^{p}
$$

and (4.9) follows. Combining the above result with estimates (4.13) and (4.14) we conclude estimate (4.10).

Using the above lemma we conclude the following.

Corollary 4.3 For given $t_{*}>0$ and function $f \in C^{1}\left[0, t_{*}\right]$ we have the following: 
i) if $k \in \mathbb{T}$ and $a \in \mathbb{R}$ are such such that $-a \neq \omega(k)$ then,

$$
\lim _{\epsilon \rightarrow 0+} \mathbb{E}\left|\sup _{t \in\left[0, t_{*}\right]} \int_{0}^{t} \exp \left\{-i \frac{a s}{\epsilon}\right\} f(s) \hat{\psi}_{\epsilon}(s, k) d s\right|=0,
$$

ii) if $k, k^{\prime} \in \mathbb{T}$ and $a \in \mathbb{R}$ are such that $-a \neq \omega(k)+\omega\left(k^{\prime}\right)$ then,

$$
\lim _{\epsilon \rightarrow 0+} \mathbb{E}\left\{\sup _{t \in\left[0, t_{*}\right]}\left|\int_{0}^{t} \exp \left\{-i \frac{a s}{\epsilon}\right\} f(s) \hat{\psi}^{(\epsilon)}(s, k) \hat{\psi}^{(\epsilon)}\left(s, k^{\prime}\right) d s\right|\right\}=0,
$$

iii) if $\omega(k)+a \neq \omega\left(k^{\prime}\right)$ then,

$$
\lim _{\epsilon \rightarrow 0+} \mathbb{E}\left\{\sup _{t \in\left[0, t_{*}\right]}\left|\int_{0}^{t} \exp \left\{-i \frac{a s}{\epsilon}\right\} f(s) \hat{\psi}^{(\epsilon)}(s, k)\left(\hat{\psi}^{(\epsilon)}\right)^{*}\left(s, k^{\prime}\right) d s\right|\right\}=0 .
$$

Proof. Using (2.12) we obtain

$$
\begin{aligned}
& \exp \left\{-i \frac{a t}{\epsilon}\right\} f(t) \hat{\psi}_{\epsilon}(t, k)-f(0) \hat{\psi}(k)=-i \frac{a+\omega(k)}{\epsilon} \int_{0}^{t} \exp \left\{-i \frac{s a}{\epsilon}\right\} f(s) \hat{\psi}_{\epsilon}(s, k) d s \\
& +\int_{0}^{t} \mathcal{P}\left[\hat{\psi}_{\epsilon}(s),\left(\hat{\psi}_{\epsilon}\right)^{*}(s)\right](k) d s+\int_{0}^{t} \sum_{y \in \mathbb{Z}} \mathcal{Q}_{y}\left[\hat{\psi}_{\epsilon}(s),\left(\hat{\psi}_{\epsilon}\right)^{*}(s)\right](k) w_{y}(d s),
\end{aligned}
$$

where $\mathcal{P}, \mathcal{Q}_{y}$ are first degree polynomials in $\hat{\psi}_{\epsilon}(s),\left(\hat{\psi}_{\epsilon}\right)^{*}(s)$ with bounded coefficients. Using Lemma 4.2 we have

$$
\mathbb{E}\left[\sup _{s \in\left[0, t_{*}\right]} \sum_{y \in \mathbb{Z}}\left|\mathcal{Q}_{y}\left[\hat{\psi}_{\epsilon}(s),\left(\hat{\psi}_{\epsilon}\right)^{*}(s)\right](k)\right|^{2}\right] \leq C\|\hat{\psi}\|_{L^{2}(\mathbb{T})}^{2} .
$$

Dividing both sides of (4.21) by $(\omega(k)+a) / \epsilon$ (possible since this factor is not equal to 0 ) we calculate

$$
\int_{0}^{t} \exp \left\{-i \frac{s a}{\epsilon}\right\} f(s) \hat{\psi}_{\epsilon}(s, k) d s .
$$

Using Lemma 4.2 we can easily conclude (4.18).

The proofs of (4.19) and (4.20) are analogous. We use the Itô formula to express $d\left[\hat{\psi}^{(\epsilon)}(s, k) \hat{\psi}^{(\epsilon)}\left(s, k^{\prime}\right)\right]$ and $d\left[\hat{\psi}^{(\epsilon)}(s, k)\left(\hat{\psi}^{(\epsilon)}\right)^{*}\left(s, k^{\prime}\right)\right]$. Then, we repeat the argument used above.

The following lemma shall be crucial for us.

Lemma 4.4 For any $f \in L^{2}(\mathbb{T}), t_{*}>0$ we have

$$
\lim _{\epsilon \rightarrow 0+} \mathbb{E}\left[\sup _{t \in\left[0, t_{*}\right]}\left|\left\langle\mathcal{M}_{t}^{(\epsilon)}, f\right\rangle\right|^{2}\right]=0
$$

and

$$
\lim _{\epsilon \rightarrow 0+} \mathbb{E}\left[\sup _{t \in\left[0, t_{*}\right]}\left|\left\langle\mathcal{N}_{t}^{(i, \epsilon)}, f\right\rangle\right|^{2}\right]=0, \quad i=1,2
$$


Proof. We only prove (4.22), the argument for (4.23) is very similar. We write

$$
\begin{aligned}
& \mathbb{E}\left|\left\langle\mathcal{M}_{t}^{(\epsilon)}, f\right\rangle\right|^{2} \leq 2\left\{\sum_{y \in \mathbb{Z}} \int_{0}^{t} d s \mathbb{E}\left|\int_{\mathbb{T}^{2}} r\left(k, k^{\prime}\right) f^{*}(k)\left(\hat{\psi}^{(\epsilon)}\right)^{*}\left(s, k-k^{\prime}\right) e_{y}^{*}\left(k^{\prime}\right) \hat{\psi}^{(\epsilon)}(s, k) d \mathbf{k}\right|^{2}\right. \\
& \left.+\sum_{y \in \mathbb{Z}} \int_{0}^{t} d s \mathbb{E}\left|\int_{\mathbb{T}^{2}} r\left(k, k^{\prime}\right) f^{*}(k)\left(\hat{\psi}^{(\epsilon)}\right)\left(s, k^{\prime}-k\right) e_{y}^{*}\left(k^{\prime}\right) \hat{\psi}^{(\epsilon)}(s, k) d \mathbf{k}\right|^{2}\right\} .
\end{aligned}
$$

Here, for abbreviation sake, we wrote $d \mathbf{k}=d k d k^{\prime}$. Using the Parseval identity we can further transform the right hand side of (4.24) into

$$
\begin{aligned}
& 2 \int_{0}^{t} d s \int_{\mathbb{T}^{3}} r\left(k, k^{\prime}\right) r\left(k_{1}, k^{\prime}\right) f^{*}(k) f\left(k_{1}\right) \\
& \times\left\{\mathbb{E}\left[\left(\hat{\psi}^{(\epsilon)}\right)^{*}\left(s, k-k^{\prime}\right) \hat{\psi}^{(\epsilon)}(s, k) \hat{\psi}^{(\epsilon)}\left(s, k_{1}-k^{\prime}\right)\left(\hat{\psi}^{(\epsilon)}\right)^{*}\left(s, k_{1}\right)\right]\right. \\
& \left.+\mathbb{E}\left[\hat{\psi}^{(\epsilon)}\left(s, k-k^{\prime}\right) \hat{\psi}^{(\epsilon)}(s, k)\left(\hat{\psi}^{(\epsilon)}\right)^{*}\left(s, k_{1}-k^{\prime}\right)\left(\hat{\psi}^{(\epsilon)}\right)^{*}\left(s, k_{1}\right)\right]\right\} d \mathbf{k},
\end{aligned}
$$

where $d \mathbf{k}=d k d k_{1} d k^{\prime}$.

Consider the term of (4.25) corresponding to the first expectation (the other can be dealt with in a similar fashion). Let

$$
\mathcal{K}_{1}=\left[\left(k, k^{\prime}, k_{1}\right): \omega(k)+\omega\left(k^{\prime}-k_{1}\right)=\omega\left(k^{\prime}\right)+\omega\left(k-k_{1}\right)\right] .
$$

Thanks to condition $\omega$ ) the three dimensional Lebesgue measure on $\mathbb{T}^{3}$ of the set vanishes. We claim that for $\mathbf{k}=\left(k, k^{\prime}, k_{1}\right) \notin \mathcal{K}_{1}$ we have

$$
\lim _{\epsilon \rightarrow 0+} \int_{0}^{t} \Psi^{(\epsilon)}(s, \mathbf{k}) d s=0,
$$

where

$$
\Psi^{(\epsilon)}(s, \mathbf{k}):=\mathbb{E}\left[\left(\hat{\psi}^{(\epsilon)}\right)^{*}\left(s, k-k^{\prime}\right) \hat{\psi}^{(\epsilon)}\left(s, k_{1}-k^{\prime}\right) \hat{\psi}^{(\epsilon)}(s, k)\left(\hat{\psi}^{(\epsilon)}\right)^{*}\left(s, k_{1}\right)\right] .
$$

Using (2.12) and Itô formula we conclude that

$$
\begin{aligned}
& \frac{i}{\epsilon}\left[\omega\left(k-k^{\prime}\right)+\omega\left(k_{1}\right)-\omega\left(k_{1}-k^{\prime}\right)-\omega(k)\right] \int_{0}^{t} \Psi^{(\epsilon)}(s, \mathbf{k}) d s \\
& =\Psi^{(\epsilon)}(t, \mathbf{k})-\Psi^{(\epsilon)}(0, \mathbf{k})+\int_{0}^{t} \mathcal{P}\left[\hat{\psi}^{(\epsilon)}(s),\left(\hat{\psi}^{(\epsilon)}\right)^{*}(s)\right](\mathbf{k}) d s
\end{aligned}
$$

where $\mathcal{P}$ is a fourth degree polynomial formed over the wave function $\hat{\psi}^{(\epsilon)}(s),\left(\hat{\psi}^{(\epsilon)}\right)^{*}(s)$. Dividing both sides of (4.27) by the factor in front of the integral on the left hand side and subsequently using (4.10) with $p=4$ we conclude (4.26). The lemma then follows, provided we can substantiate the following interchange of the limit with integral

$$
\begin{aligned}
& \lim _{\epsilon \rightarrow 0+} \int_{0}^{t} d s \int_{\mathbb{T}^{3}} r\left(k, k^{\prime}\right) r\left(k_{1}, k^{\prime}\right) f^{*}(k) f\left(k_{1}\right) \Psi^{(\epsilon)}(s, \mathbf{k}) d \mathbf{k} \\
& =\int_{\mathbb{T}^{3}} r\left(k, k^{\prime}\right) r\left(k_{1}, k^{\prime}\right) f^{*}(k) f\left(k_{1}\right) d \mathbf{k}\left\{\lim _{\epsilon \rightarrow 0+} \int_{0}^{t} \Psi^{(\epsilon)}(s, \mathbf{k}) d s\right\} .
\end{aligned}
$$

The latter however is a consequence of the Lebesgue dominated convergence theorem and (4.10). This ends the proof of (4.22). The proof of (4.23) is analogous. 


\section{Proof of Proposition 4.1}

We first demonstrate (4.8). It is a consequence of parts ii) and iii) of Corollary 4.3, and the Lebesgue dominated convergence theorem. Indeed,

$$
\mathbb{E}\left\{\sup _{t \in\left[0, t_{*}\right]}\left|\mathcal{V}_{\epsilon, a}^{(1)}(t)\right|\right\} \leq \mathbb{E}\left\{\int_{\mathbb{T}} d k \sup _{t \in\left[0, t_{*}\right]}\left|\int_{0}^{t} \exp \left\{\frac{i s a}{\epsilon}\right\} \hat{\psi}^{(\epsilon)}(s, k) \hat{\psi}^{(\epsilon)}(s,-k) f^{*}(k) d s\right|\right\} .
$$

Using condition $\omega$ ) we conclude that the expression under the integral over $k$ on the right hand side vanishes, as $\epsilon \rightarrow 0+$, possibly outside a set of $k$-s of null Lebesgue measure. Invoking again (4.10) we can substantiate exchanging of taking the limit and integration and (4.8) follows.

As for (4.7), observe that from the Itô formula for $d\left|\hat{\psi}^{(\epsilon)}(t, k)\right|^{2}$ we have

$$
\left\langle\left|\hat{\psi}^{(\epsilon)}(t)\right|^{2}, f\right\rangle-\left\langle|\hat{\psi}(0)|^{2}, f\right\rangle=\int_{0}^{t}\left\langle\mathcal{L}\left|\hat{\psi}^{(\epsilon)}(s)\right|^{2}, f\right\rangle d s+\frac{1}{2} \int_{0}^{t}\left\langle\mathcal{L} \hat{\psi}_{2}^{(\epsilon)}(s), f\right\rangle d s+\left\langle\mathcal{M}_{t}^{(\epsilon)}, f\right\rangle .
$$

Denote by $\left\{Q_{\epsilon}, \epsilon \in(0,1]\right\}$ the family of the laws of $\left\{\left|\hat{\psi}^{(\epsilon)}(t)\right|^{2}, t \geq 0\right\}$ over $C\left([0,+\infty), L_{w}^{2}(\mathbb{T})\right)$. Here $L_{w}^{2}(\mathbb{T})$ stands for the space $L^{2}(\mathbb{T})$ equipped with the weak topology.

Using Lemma 4.2 we conclude from the above equality that for any $t_{*}>0$ there exists a constant $C>0$ such that

$$
\mathbb{E}\left|\left\langle\left|\hat{\psi}^{(\epsilon)}(t)\right|^{2}, f\right\rangle-\left\langle\left|\hat{\psi}^{(\epsilon)}(s)\right|^{2}, f\right\rangle\right|^{4} \leq C(t-s)^{2}, \quad \forall \epsilon \in(0,1], t, s \in\left[0, t_{*}\right] .
$$

This, according to Theorem 12. 3 of [6], implies tightness of the family of the laws of $\left\{\left\langle\left|\hat{\psi}^{(\epsilon)}(t)\right|^{2}, f\right\rangle, t \geq 0\right\}$, as $\epsilon \rightarrow 0+$, over $C[0,+\infty)$ equipped with the usual topology of uniform convergence on compact intervals. From the above and estimate (4.9) we conclude weak precompactness of $Q_{\epsilon}, \epsilon \in(0,1]$, see Theorem 3.1, p. 276 of [10]. Thanks to Lemma 4.4 and the already proved formula (4.8) we conclude that the limiting law is a $\delta$-type measure supported on $\overline{\mathcal{E}}(t)$ - the solution of (3.4). This, in particular, implies that

$$
\lim _{\epsilon \rightarrow 0+} \sup _{t \in\left[0, t_{*}\right]}\left|\left\langle\left|\hat{\psi}^{(\epsilon)}(t)\right|^{2}-\overline{\mathcal{E}}(t), f\right\rangle\right|=0
$$

in probability. Hence (4.7) follows.

\section{Proof of part (i) of Theorem 3.1}

With the results proved above in hand, we return to the proof of part (i) Theorem 3.1. Assume first that $n=1$ and we consider the process $\tilde{\psi}^{(\epsilon)}(t, k)$ evaluated at a single $k$. From (2.17) and (4.10) we conclude easily that for any $t_{*}>0$ there exists a constant $C>0$ such that

$$
\mathbb{E}\left|\tilde{\psi}^{(\epsilon)}(t, k)-\tilde{\psi}^{(\epsilon)}(s, k)\right|^{4} \leq C(t-s)^{2}, \quad \forall \epsilon \in(0,1], s, t \in\left[0, t_{*}\right] .
$$

This implies tightness of the laws of $\left\{\tilde{\psi}^{(\epsilon)}(t, k), t \geq 0\right\}$ over $C[0,+\infty)$.

In the next step we identify the limiting law $P_{k}$ of $\left\{\tilde{\psi}^{(\epsilon)}(t, k), t \geq 0\right\}$ over $C[0,+\infty)$. Denote by $\Pi_{t}(f):=f(t), f \in C[0,+\infty)$ the canonical coordinate map.

Consider the complex valued martingale given by (2.19). Its quadratic variation is given by (4.2) and, of course, $\left\langle\tilde{\mathcal{M}}^{(\epsilon)}(k), \tilde{\mathcal{M}}^{(\epsilon)}(k)\right\rangle_{t}=0$. Using Proposition 4.1 we conclude that

$$
\lim _{\epsilon \rightarrow 0+} \sup _{t \in\left[0, t_{*}\right]}\left|\left\langle\tilde{\mathcal{M}}^{(\epsilon)}(k),\left(\tilde{\mathcal{M}}^{(\epsilon)}\right)^{*}(k)\right\rangle_{t}-\int_{0}^{t} \mathcal{R}(s, k) d s\right|=0 .
$$


Then by virtue of Theorem 5.4 of $[9]$ we conclude that $\left\{\tilde{\mathcal{M}}_{t}^{(\epsilon)}, t \geq 0\right\}$ converge in law over $C[0,+\infty)$ to a complex valued Gaussian process $\left\{\tilde{\mathcal{M}}_{t}, t \geq 0\right\}$ given by

$$
\tilde{\mathcal{M}}_{t}(k):=\int_{0}^{t} \mathcal{R}^{1 / 2}(s, k) w(d s),
$$

where $\{w(t), t \geq 0\}$ is a complex valued standard Brownian motion.

Assume now that $k \neq 0$ and $P_{k}$ is a limiting law of $\left\{\tilde{\psi}^{(\epsilon)}(t, k), t \geq 0\right\}$ obtained from a certain sequence $\epsilon_{n} \rightarrow 0+$. Denote by $\Pi_{t}$ the coordinate mapping, given by $\Pi_{t}(g):=g(t)$ for $g \in C[0,+\infty)$. From (2.17) and (4.18) we infer that

$$
\Pi_{t}+\frac{\hat{\beta}(k)}{4} \int_{0}^{t} \Pi_{s} d s, \quad t \geq 0
$$

is a $P_{k}$-martingale whose law coincides with that of the process described by (4.28). The conclusion extends also to the case when $k=0$ and $\omega(0)>0$. If, on the other hand, $\omega(0)=0$ we have $\hat{\beta}(0)=0$ and $\mathcal{R}^{1 / 2}(s, 0)=0$ and therefore $\Pi_{t} \equiv \Pi_{0}$ a.s.

Suppose now that $k_{1}, \ldots, k_{n} \in \mathbb{T}$ are pairwise distinct. Denote by $Q_{\epsilon}$ the law of

$$
\left\{\left(\tilde{\psi}^{(\epsilon)}\left(t, k_{1}\right), \ldots, \tilde{\psi}^{(\epsilon)}\left(t, k_{n}\right)\right), t \geq 0\right\}
$$

over $C\left([0,+\infty), \mathbb{C}^{n}\right)$. Then, we claim that

$$
\lim _{\epsilon \rightarrow 0+} \sup _{t \in\left[0, t_{*}\right]}\left|\left\langle\tilde{\mathcal{M}}^{(\epsilon)}\left(k_{i}\right),\left(\tilde{\mathcal{M}}^{(\epsilon)}\right)^{*}\left(k_{j}\right)\right\rangle_{t}-\delta_{i, j} \int_{0}^{t} \mathcal{R}\left(s, k_{i}\right) d s\right|=0
$$

and, obviously,

$$
\lim _{\epsilon \rightarrow 0+} \sup _{t \in\left[0, t_{*}\right]}\left|\left\langle\tilde{\mathcal{M}}^{(\epsilon)}\left(k_{i}\right), \tilde{\mathcal{M}}^{(\epsilon)}\left(k_{j}\right)\right\rangle_{t}\right|=0, \quad \forall i, j=1, \ldots, n
$$

To see (4.29) note that for $i \neq j$ we have

$$
\begin{aligned}
& \left\langle\tilde{\mathcal{M}}^{(\epsilon)}\left(k_{i}\right),\left(\tilde{\mathcal{M}}^{(\epsilon)}\right)^{*}\left(k_{j}\right)\right\rangle_{t}=\sum_{\sigma_{1}, \sigma_{2}= \pm 1} \sigma_{1} \sigma_{2} \int_{0}^{t} \exp \left\{\frac{i\left(\omega\left(k_{i}\right)-\omega\left(k_{j}\right)\right) s}{\epsilon}\right\} d s \\
& \times \int_{\mathbb{T}} r\left(k_{i}, k^{\prime}\right) r\left(k_{j}, k^{\prime}\right) \hat{\psi}_{\sigma_{1}}^{(\epsilon)}\left(s, k_{i}-k^{\prime}\right)\left(\hat{\psi}_{\sigma_{2}}^{(\epsilon)}\right)^{*}\left(s, k_{j}-k^{\prime}\right) d k^{\prime}
\end{aligned}
$$

Using part iii) of Corollary 4.3 combined with condition $\omega$ ) we conclude, thanks to the fact that $k_{i} \neq k_{j}$, that

$$
\lim _{\epsilon \rightarrow 0+} \sup _{t \in\left[0, t_{*}\right]}\left|\int_{0}^{t} \exp \left\{\frac{i\left(\omega\left(k_{i}\right)-\omega\left(k_{j}\right)\right) s}{\epsilon}\right\} \hat{\psi}_{\sigma_{1}}^{(\epsilon)}\left(s, k_{i}-k^{\prime}\right)\left(\hat{\psi}_{\sigma_{2}}^{(\epsilon)}\right)^{*}\left(s, k_{j}-k^{\prime}\right) d s\right|=0
$$

for a.e. $k^{\prime} \in \mathbb{T}$. Using (4.10) in the same way as in the proof of (4.8) we can substantiate exchanging the passage to the limit with the respective integration and conclude (4.29).

Combining (4.29) and (4.30) with (4.18) we obtain from equation (2.17) that any limiting point of the family of laws of $Q_{\epsilon_{n}}$ as $\epsilon_{n} \rightarrow 0+$ is a measure $P_{k_{1}, \ldots, k_{n}}$ such that

$$
\mathcal{M}_{t}=\left(\mathcal{M}_{t}^{(1)}, \ldots, \mathcal{M}_{t}^{(n)}\right):=\Pi_{t}+\frac{\hat{\beta}(k)}{4} \int_{0}^{t} \Pi_{s} d s, \quad t \geq 0
$$


is $\mathbb{C}^{n}$-valued martingale, whose quadratic covariation is given by

$$
\left\langle\mathcal{M}^{(i)},\left(\mathcal{M}^{(j)}\right)^{*}\right\rangle_{t}=\delta_{i, j} \int_{0}^{t} \mathcal{R}\left(s, k_{j}\right) d s
$$

and

$$
\left\langle\mathcal{M}^{(i)},\left(\mathcal{M}^{(j)}\right)\right\rangle_{t}=0, \quad \forall i, j=1, \ldots, n .
$$

This of course implies that $P_{k_{1}, \ldots, k_{n}}=P_{k_{1}} \otimes \ldots \otimes P_{k_{n}}$.

\section{Proof of part ii) of Theorem 3.1}

Let $f \in L^{2}(\mathbb{T})$. We shall prove that

$$
\lim _{\epsilon \rightarrow 0+} \mathbb{E}\left|\left\langle\tilde{\mathcal{M}}_{t}^{(\epsilon)}, f\right\rangle\right|^{2}=0 .
$$

Assuming this result we show how to finish the proof of part (ii). Denote

$$
\delta \psi^{(\epsilon)}(t):=\tilde{\psi}^{(\epsilon)}(t)-\bar{\psi}(t) .
$$

Using Lemma 4.2 and Theorem 3.1, p. 276 of 10 we can conclude weak pre-compactness of $P_{\epsilon}, \epsilon \in(0,1]$ - the family of the laws of $\left\{\delta \psi^{(\epsilon)}(t), t \geq 0\right\}-$ in $C\left([0,+\infty), L_{w}^{2}(\mathbb{T})\right)$. With the help of Corollary 4.19 and (4.31) we conclude that the limiting measure, as $\epsilon \rightarrow 0+$, is supported on the solution of the equation

$$
\langle g(t), f\rangle-\frac{1}{4} \int_{0}^{t}\langle\hat{\beta} g(s), f\rangle d s=0, \quad \forall f \in L^{2}(\mathbb{T}) .
$$

This of course shows that it is the $\delta$-measure supported on $g(t) \equiv 0$. Hence, in particular we get

$$
\lim _{\epsilon \rightarrow 0+} \sup _{t \in\left[0, t_{*}\right]}\left|\left\langle\delta \psi^{(\epsilon)}(t), f\right\rangle\right|=0
$$

in probability and (3.6) follows.

Coming back to the proof of (4.31) note that by the definition of the martingale $\tilde{\mathcal{M}}_{t}^{(\epsilon)}$, see (2.19), we only need to show that

$$
\lim _{\epsilon \rightarrow 0+} \mathbb{E}\left|\int_{0}^{t} \int_{\mathbb{T}^{2}} \exp \left\{i s \frac{\omega(k)}{\epsilon}\right\} r\left(k, k^{\prime}\right) f^{*}(k) \hat{\psi}_{\sigma}^{(\epsilon)}\left(s, k-k^{\prime}\right) d W\left(s, k^{\prime}\right) d k\right|^{2}=0
$$

for $\sigma= \pm 1$. We consider only the case $\sigma=1$, the other one can be dealt in a similar manner. The expression under the limit in (4.33) equals

$$
\int_{0}^{t} \int_{\mathbb{T}^{3}} \exp \left[i s \frac{\omega(k)-\omega\left(k_{1}\right)}{\epsilon}\right] r\left(k, k^{\prime}\right) r\left(k_{1}, k^{\prime}\right) f^{*}(k) f\left(k_{1}\right) \mathbb{E}\left[\hat{\psi}^{(\epsilon)}\left(s, k-k^{\prime}\right)\left(\hat{\psi}^{(\epsilon)}\right)^{*}\left(s, k_{1}-k^{\prime}\right)\right] d s d \mathbf{k},
$$

with $d \mathbf{k}=d k d k_{1} d k^{\prime}$. Using Corollary 4.3 and an argument identical with the one used in the proof of Lemma 4.4 we conclude that

$$
\lim _{\epsilon \rightarrow 0+} \int_{0}^{t} \exp \left\{i s \frac{\omega(k)-\omega\left(k_{1}\right)}{\epsilon}\right\} \mathbb{E}\left[\hat{\psi}^{(\epsilon)}\left(s, k-k^{\prime}\right)\left(\hat{\psi}^{(\epsilon)}\right)^{*}\left(s, k_{1}-k^{\prime}\right)\right] d s=0
$$

for all $k^{\prime}, k, k_{1}$ such that $\omega\left(k-k^{\prime}\right)+\omega\left(k_{1}\right)-\omega(k) \neq \omega\left(k_{1}-k^{\prime}\right)$. Since the latter inequality holds on the set of null Lebesgue measure we conclude equality in (4.33), thanks to the Lebesgue dominated convergence theorem. 


\section{Spatially homogeneous initial data}

Tightness of the family of laws $\left\{\tilde{\psi}^{(\epsilon)}(t), t \geq 0\right\}$, in the space of continuous functionals taking values in a space of distributions is again due to the fact that the evolution equation (2.17) contains no terms that are large in magnitude. This is done in Sections 5.1 and 5.2 , However, we have no estimates of the $H^{-m}(\mathbb{T})$ norm of $\tilde{\psi}^{(\epsilon)}(t)$ analogous to the ones in Lemma 4.2, that have played an important role in the limit identification argument of Section 4 for square integrable data. Therefore, instead of considering the quadratic variation of the martingale term as we did in the proof of Theorem 3.1, for the proof of Theorem 3.3 we identify the limit of all moments of $\tilde{\psi}^{(\epsilon)}(t)$. Accordingly, we first write equations for time evolution of an arbitrary moment of $\tilde{\psi}^{(\epsilon)}(t)$ in Section 5.3. Using standard averaging argument we show (see Proposition 5.3) the convergence of moments, as $\epsilon \rightarrow 0+$, to a solution of the limiting equation obtained simply by discarding the oscillatory terms from the moment equation. Finally in Section 5.5 we prove that the solutions of the limiting equation coincide with the respective moments of the non-homogeneous Ornstein-Uhlenbeck equation (3.17) concluding in this way the proof of Theorem 3.3 .

\subsection{Properties of spatially homogeneous solutions of (2.12)}

The initial data $\hat{\psi}$ considered in this section is random and takes values in the Hilbert space of distributions $H^{-m}(\mathbb{T})$ for some $m>1 / 2$. In fact, in Sections 5.15 .4 we shall not make any use of the assumption that the data is Gaussian and we use only the fact that it is spatially homogeneous and

$$
\mathbb{E}\|\hat{\psi}\|_{H^{-m}(\mathbb{T})}^{2}<+\infty .
$$

Gaussianity shall be used only in Section 5.5.

Consider the random field $\left\{\psi_{y}:=\left\langle\hat{\psi}, e_{y}\right\rangle, y \in \mathbb{Z}\right\}$. The field is assumed to be spatially homogeneous, i.e. $\left\{\psi_{y+z}, y \in \mathbb{Z}\right\}$ and $\left\{\psi_{y}, y \in \mathbb{Z}\right\}$ have identical laws for all $z \in \mathbb{Z}$, and centered, i.e. $\mathbb{E} \psi_{0}=0$. Spatial homogeneity is equivalent to the fact that $\hat{\psi}(k)$ and $e_{z}(k) \hat{\psi}(k)$ are identically distributed in $H^{-m}(\mathbb{T})$ for any $z \in \mathbb{Z}$. Note that, since $m>1 / 2$,

$$
\sum_{y \in \mathbb{Z}}\left(1+y^{2}\right)^{-m} \mathbb{E}\left|\psi_{y}\right|^{2}=\mathbb{E}\|\hat{\psi}\|_{H^{-m}(\mathbb{T})}^{2}<+\infty
$$

due to (5.1).

Since the covariance function of the field

$$
S_{x-y}:=\mathbb{E}\left[\psi_{x} \psi_{y}^{*}\right], \quad \forall x, y \in \mathbb{Z}
$$

is positive definite, there exists a finite measure $\hat{E}(d k)$ such that

$$
S_{x}=\int_{\mathbb{T}} e^{i x k} \hat{E}(d k), \quad \forall x \in \mathbb{Z}
$$

We assume that the covariance function decays sufficiently fast in space so that

$$
\sum_{x \in \mathbb{Z}}\left(\left|\mathbb{E}\left[\psi_{x}^{*} \psi_{0}\right]\right|+\left|\mathbb{E}\left[\psi_{x} \psi_{0}\right]\right|\right)<+\infty
$$


Assumption (5.2) implies, in particular, that $\hat{E}(d k)=\mathcal{E}_{0}(k) d k$ for some non-negative energy density $\mathcal{E}_{0} \in C(\mathbb{T})$ and both this function and $\mathcal{Y}=\sum_{x \in \mathbb{Z}} e_{x} \mathbb{E}\left[\psi_{x} \psi_{0}\right]$ belong to $C(\mathbb{T})$. When the field $\psi_{x}$ is a complex valued Gaussian, as described Section 3.2 , we have $\mathcal{Y} \equiv 0$. This and (3.14) together imply (5.2).

We note that the translation invariance of the solution persists in time. Indeed, let $\psi_{x}^{(\epsilon)}(t):=\left\langle\hat{\psi}^{(\epsilon)}(t), e_{x}\right\rangle$ and $z \in \mathbb{Z}$. A direct computation shows that $e_{z} \hat{\psi}^{(\epsilon)}(t)$ is also a solution of (2.17). Since the laws of the initial conditions $e_{z} \hat{\psi}$ and that of $\hat{\psi}$ are identical, we conclude from the uniqueness in law of solutions that the same holds for the processes $\left\{e_{z} \hat{\psi}^{(\epsilon)}(t), t \geq 0\right\}$ and $\left\{\hat{\psi}^{(\epsilon)}(t), t \geq 0\right\}$. In consequence, the laws of $\left\{\psi_{x}^{(\epsilon)}(t), x \in \mathbb{Z}\right\}$ and that of $\left\{\psi_{x+z}^{(\epsilon)}(t), x \in \mathbb{Z}\right\}$ are identical for any $z \in \mathbb{Z}$. We can now define the correlation functions

$$
S_{t, x}^{(\epsilon)}=\mathbb{E}\left[\psi_{x}^{(\epsilon)}(t)\left(\psi_{0}^{(\epsilon)}\right)^{*}(t)\right] \quad \text { and } \quad Y_{t, x}^{(\epsilon)}=\mathbb{E}\left[\psi_{x}^{(\epsilon)}(t) \psi_{0}^{(\epsilon)}(t)\right]
$$

and introduce two distributions on $H^{-m}(\mathbb{T})$

$$
\left\langle f, \hat{S}_{t}^{(\epsilon)}\right\rangle:=\sum_{x \in \mathbb{Z}} \check{f}_{x}\left(S_{t, x}^{(\epsilon)}\right)^{*} \quad \text { and } \quad\left\langle f, \hat{Y}_{t}^{(\epsilon)}\right\rangle:=\sum_{x \in \mathbb{Z}} \check{f}_{x}\left(Y_{t, x}^{(\epsilon)}\right)^{*} .
$$

We recall the following result of [4].

Proposition 5.1 For any $\epsilon \in(0,1]$ and $t \geq 0$ we have $\hat{S}_{t}^{(\epsilon)}, \hat{Y}_{t}^{(\epsilon)} \in L^{1}(\mathbb{T})$. Moreover,

(1) $\hat{S}_{t}^{(\epsilon)}$ is non-negative, and for any $t_{*}>0$

$$
\sup _{\epsilon \in(0,1]} \sup _{t \in\left[0, t_{*}\right]}\left(\left\|\hat{S}_{t}^{(\epsilon)}\right\|_{L^{1}(\mathbb{T})}+\left\|\hat{Y}_{t}^{(\epsilon)}\right\|_{L^{1}(\mathbb{T})}\right)<+\infty,
$$

(2) for any $f \in L^{\infty}(\mathbb{T})$ we have

$$
\lim _{\epsilon \rightarrow 0+} \sup _{t \in\left[0, t_{*}\right]}\left|\left\langle\hat{S}_{t}^{(\epsilon)}-\overline{\mathcal{E}}(t), f\right\rangle\right|=0,
$$

where $\overline{\mathcal{E}}(t)$ is given by (3.4) with the initial condition replaced by $\mathcal{E}_{0}(k)$

(3) for any $f$ such that $f \omega^{-1} \in L^{\infty}(\mathbb{T})$ we have

$$
\lim _{\epsilon \rightarrow 0+} \sup _{t \in\left[0, t_{*}\right]}\left|\int_{0}^{t}\left\langle\hat{Y}_{s}^{(\epsilon)}, f\right\rangle d s\right|=0 .
$$

Proof. Parts 1) and 2) of the lemma are contained in Lemma 12 and Theorem 10 of 4], respectively. Part 3) follows easily from part 1) and the arguments used in the proof of Corollary 4.3 ,

\subsection{Tightness of solutions of (2.17)}

Given $f \in H^{m}(\mathbb{T})$, we denote by $Q_{\epsilon}$ and $Q_{\epsilon, f}$ the laws of the processes $\left\{\hat{\psi}^{(\epsilon)}(t), t \geq 0\right\}$ and $\left\{\left\langle f, \hat{\psi}^{(\epsilon)}(t)\right\rangle, t \geq 0\right\}$ over $C\left([0,+\infty), H_{w}^{-m}(\mathbb{T})\right)$ and $C([0,+\infty), \mathbb{C})$, respectively, and by $\left\{\tilde{Q}_{\epsilon}, \epsilon \in(0,1]\right\}$ the family of laws of $\left\{\tilde{\psi}^{(\epsilon)}(t), t \geq 0\right\}$ over $C\left([0,+\infty), H_{w}^{-m}(\mathbb{T})\right)$. According to [17, see Remark R1, p. 997, to verify the tightness of $\tilde{Q}_{\epsilon}$, it suffices to show the following two conditions: 
(UC) for any $\sigma, M, t_{*}>0$ there exists a $\delta>0$ such that

$$
\mathbb{P}\left[\sup _{t \in\left[0, t_{*}\right]}\left|\left\langle\tilde{\psi}^{(\epsilon)}(t), f\right\rangle\right| \geq M\right]<\sigma, \quad \forall\|f\|_{H^{m}(\mathbb{T})}<\delta, \quad \epsilon \in(0,1],
$$

and

(FDT) for any $f \in H^{m}(\mathbb{T})$ the family of the laws of the processes $\left\{\left\langle\tilde{\psi}^{(\epsilon)}(t), f\right\rangle, t \in\left[0, t_{*}\right]\right\}$, $\epsilon \in(0,1]$ is tight over $C\left[0, t_{*}\right]$ for any $t_{*}>0$.

As in (3.16) we conclude that for any $f_{1}, f_{2} \in H^{m}(\mathbb{T})$, where $m>1 / 2$, the covariance

$$
\mathbb{E}\left[\left\langle f_{1}, \hat{\psi}_{t}^{(\epsilon)}\right\rangle\left\langle f_{2}, \hat{\psi}_{t}^{(\epsilon)}\right\rangle^{*}\right]=\int_{\mathbb{T}} \hat{S}_{t}^{(\epsilon)}(k) f_{1}(k) f_{2}^{*}(k) d k .
$$

From (2.17) and Doob's inequality there exists a constant $C>0$ such that

$\mathbb{E}\left[\sup _{t \in\left[0, t_{*}\right]}\left|\left\langle\tilde{\psi}^{(\epsilon)}(t), f\right\rangle\right|^{2}\right] \leq C\left\{\mathbb{E}|\langle\hat{\psi}, f\rangle|^{2}+\int_{0}^{t_{*}} \mathbb{E}\left|\left\langle\mathcal{A}\left[\frac{t}{\epsilon}, \tilde{\psi}^{(\epsilon)}(t)\right], f\right\rangle\right|^{2} d t+\mathbb{E}\left|\left\langle\tilde{\mathcal{M}}_{t_{*}}^{(\epsilon)}, f\right\rangle\right|^{2}\right\}$.

Using (5.6), (5.3) and the definitions of $\mathcal{A}[t / \epsilon, \cdot]$, and the martingale $\tilde{\mathcal{M}}_{t}^{(\epsilon)}$ (see (2.18) and (2.19) ) we conclude that the right hand side of (5.7) can be estimated from above by $C\|f\|_{\infty}^{2}$, which can be made less than $\sigma>0$, provided we choose $\delta>0$ sufficiently small.

To show condition (FDT) consider $\tilde{Q}_{\epsilon, f}^{(M)}$ - the law of the stopped process

$$
\left\{\left(\left\langle\tilde{\psi}^{(\epsilon)}\left(t \wedge \tau_{M}^{(\epsilon)}\right), f\right\rangle,\left\langle\tilde{\psi}^{(\epsilon)}\left(t \wedge \tau_{M}^{(\epsilon)}\right), f_{0}\right\rangle\right) t \in\left[0, t_{*}\right]\right\}
$$

over $C\left(\left[0, t_{*}\right] ; \mathbb{C}^{2}\right)$. Here $f_{0}(k):=f(-k)$ and

$$
\tau_{M}^{(\epsilon)}:=\inf \left[t \in\left[0, t_{*}\right]:\left|\left\langle\tilde{\psi}^{(\epsilon)}(t), f\right\rangle\right|^{2}+\left|\left\langle\tilde{\psi}^{(\epsilon)}(t), f_{0}\right\rangle\right|^{2} \geq M^{2}\right] .
$$

We adopt the convention that $\tau_{M}:=t_{*}$ if the set is empty. Thanks to (UC) we conclude that $\lim _{M \rightarrow+\infty} \tau_{M}^{(\epsilon)}=t_{*}$, a.s. for each $\epsilon \in(0,1]$. Denote also by $\tilde{Q}_{\epsilon, f}$ the law of the process without the stopping condition.

From (2.17) we conclude that for a fixed $M$ and an arbitrary non-negative function $\phi$ : $\mathbb{C}^{2} \rightarrow \mathbb{R}$, of class $C_{c}^{1}\left(\mathbb{R}^{4}\right)$, one can choose a constant $K_{\phi}$, independent of spatial translations of $\phi$, such that

$$
\phi\left(\left\langle\tilde{\psi}^{(\epsilon)}\left(t \wedge \tau_{M}^{(\epsilon)}\right), f\right\rangle,\left\langle\tilde{\psi}^{(\epsilon)}\left(t \wedge \tau_{M}^{(\epsilon)}\right), f_{0}\right\rangle\right)+K_{\phi} t, t \in\left[0, t_{*}\right]
$$

is a non-negative submartingale. This proves tightness of $\left\{\tilde{Q}_{\epsilon, f}^{(M)}, \epsilon \in(0,1]\right\}$ for a fixed $M$, by virtue of Theorem 1.4.3 of [19]. Since for any $\sigma>0$ one can find a sufficiently large $M>0$ such that $B_{M}$ - the ball centered at 0 and of radius $M$ in $C\left(\left[0, t_{*}\right] ; \mathbb{C}^{2}\right)$ - satisfies

$$
\tilde{Q}_{\epsilon, f}^{(M)}\left(B_{M}^{c}\right)+\tilde{Q}_{\epsilon, f}\left(B_{M}^{c}\right)<\sigma
$$

and

$$
\tilde{Q}_{\epsilon, f}^{(M)}\left(B_{M} \cap A\right)=\tilde{Q}_{\epsilon, f}\left(B_{M} \cap A\right)
$$

for all Borel measurable subsets $A$ of $C\left(\left[0, t_{*}\right] ; \mathbb{C}^{2}\right)$, we conclude tightness of $\left\{\tilde{Q}_{\epsilon, f}, \epsilon \in(0,1]\right\}$, see step (vi) of the proof of Theorem 3 of [1] for details of this argument. 


\subsection{Evolution of moments}

To describe the evolution of moments we rewrite equation (2.17) in a more compact form, as a $2 \times 2$ linear system of equations with multiplicative noise. Denote by $\mathbf{C}(t, k)=\left[C_{i j}(t, \mathbf{k})\right]$, $i, j= \pm 1$, the $2 \times 2$ hermitian matrix

$$
\mathbf{C}(t, k):=\left[\begin{array}{ll}
C_{1,1} & C_{1,-1} \\
C_{-1,1} & C_{-1,-1}
\end{array}\right]
$$

with the entries

$$
C_{p, q}(t, k):=\frac{p q \hat{\beta}(k)}{4} \exp \{i p \omega(k)(1-p q) t\} .
$$

Let also $\mathbf{Q}\left(t, k, k^{\prime}\right)=\left[Q_{p q}\left(t, k, k^{\prime}\right)\right], p, q= \pm 1$, be the $2 \times 2$ matrix

$$
Q_{p, q}\left(t, k, k^{\prime}\right):=\operatorname{ipqr}\left(k, k-k^{\prime}\right) e^{i p\left[\omega(k)-p q \omega\left(k^{\prime}\right)\right] t}
$$

and $W(t, k):=\sum_{y} e_{y}(k) w_{y}(t)$. Let us recall that $\tilde{\psi}_{-1}^{(\epsilon)}(t, k)=\tilde{\psi}^{(\epsilon) *}(t,-k)$. Then, equation for

$$
\Psi^{(\epsilon)}(t, k)=\left[\begin{array}{c}
\tilde{\psi}^{(\epsilon)}(t, k) \\
\tilde{\psi}_{-1}^{(\epsilon)}(t, k)
\end{array}\right]
$$

is

$$
\begin{aligned}
& d \Psi^{(\epsilon)}(t, k)=-\mathbf{C}\left(\frac{t}{\epsilon}, k\right) \Psi^{(\epsilon)}(t, k) d t+\int_{\mathbb{T}} \mathbf{Q}\left(\frac{t}{\epsilon}, k, k-k^{\prime}\right) \Psi^{(\epsilon)}\left(t, k-k^{\prime}\right) W\left(d t, d k^{\prime}\right), \\
& \Psi^{(\epsilon)}(0, k)=\Psi(k)
\end{aligned}
$$

with the initial data

$$
\Psi(k)=\left[\begin{array}{c}
\hat{\psi}(k) \\
\hat{\psi}_{-1}(k)
\end{array}\right] .
$$

Let $\left\{\mathbf{S}_{\epsilon}(s, t, k), s, t \in \mathbb{R}\right\}$ be the $2 \times 2$ Hermitian matrices solving the deterministic system

$$
\begin{aligned}
& \frac{d \mathbf{S}_{\epsilon}(s, t, k)}{d t}=-\mathbf{C}\left(\frac{t}{\epsilon}, k\right) \mathbf{S}_{\epsilon}(s, t, k) \\
& \mathbf{S}_{\epsilon}(s, s, k)=I_{2} .
\end{aligned}
$$

Here $I_{2}$ is the $2 \times 2$ identity matrix. Existence and uniqueness of solutions to (5.8) in the strong sense (thus implying the result in the mild, or weak sense as well) follows from an argument used in Chapter 6 of [7] (because the generators for the evolution family $\mathbf{S}_{\epsilon}(s, t)$ are bounded), see Proposition 6.4 there. Although the case considered here differs slightly because the coefficients are time dependent, this does not influence the results.

Given a nonnegative integer $p \geq 1$, define a tensor valued distribution on $H^{-m / p}\left(\mathbb{T}^{p}\right)$

$$
\hat{M}^{(\epsilon)}(t):=\left[\hat{M}_{\mathbf{i}}^{(\epsilon)}(t)\right], \quad \mathbf{i}=\left(i_{1}, \ldots, i_{p}\right) \in\{-1,1\}^{p},
$$

by

$$
\hat{M}_{\mathbf{i}}^{(\epsilon)}(t)=\mathbb{E}\left[\tilde{\psi}_{i_{1}}^{(\epsilon)}(t) \otimes \ldots \otimes \tilde{\psi}_{i_{p}}^{(\epsilon)}(t)\right]
$$


Note that also

$$
\hat{M}_{\mathbf{i}}^{(\epsilon)}(0)=\hat{M}_{\mathbf{i}}:=\mathbb{E}\left[\hat{\psi}_{i_{1}} \otimes \ldots \otimes \hat{\psi}_{i_{p}}\right]
$$

For a given multi-index $\mathbf{i}$ we define the multi-indices $\mathbf{i}_{\ell}(j)=\left(i_{1}^{\prime}, \ldots, i_{p}^{\prime}\right), \mathbf{i}_{\ell, m}\left(j_{1}, j_{2}\right)=$ $\left(i_{1}^{\prime \prime}, \ldots, i_{p}^{\prime \prime}\right)$ given by: $i_{q}^{\prime}=i_{q}$ for $q \neq \ell$ and $i_{\ell}^{\prime}=j$, and $i_{q}^{\prime \prime}=i_{q}$ for $q \neq \ell, m$ and $i_{\ell}^{\prime \prime}=j_{1}$, $i_{m}^{\prime \prime}=j_{2}$. Denote by $\mathcal{M}\left(\mathbb{T}^{p}\right)$ the space of all complex valued Borel measures $\nu$ on $\mathbb{T}^{p}$ whose total variation norm $\|\nu\|_{\mathrm{TV}}$ is finite.

Proposition 5.2 The following are true:

1) $\hat{M}^{(\epsilon)}(t)$ is the unique solution in $H^{-m / p}\left(\mathbb{T}^{p}\right)$ of the system of equations

$$
\begin{aligned}
& \frac{d}{d t} \hat{M}_{\mathbf{i}}^{(\epsilon)}(t, \mathbf{k})=-\sum_{\ell=1}^{p} \sum_{j= \pm 1} C_{i_{\ell}, j}\left(\frac{t}{\epsilon}, k_{\ell}\right) \hat{M}_{\mathbf{i}_{\ell}(j)}^{(\epsilon)}(t, \mathbf{k}) \\
& +\sum_{1 \leq \ell<m \leq p} \sum_{j_{1}, j_{2}= \pm 1} \int_{\mathbb{T}} \mathcal{R}_{i_{\ell}, i_{m}}^{j_{1}, j_{2}}\left(\frac{t}{\epsilon}, k_{\ell}, k_{m}, k^{\prime}\right) \hat{M}_{\mathbf{i}_{\ell, m}\left(j_{1}, j_{2}\right)}^{(\epsilon)}\left(t, \mathbf{k}_{\ell, m}^{\prime}\right) d k^{\prime}
\end{aligned}
$$

with $\mathbf{i} \in\{-1,1\}^{p}$ and the initial data given by (5.9). Here

$$
\mathcal{R}_{i_{\ell}, i_{m}}^{j_{1}, j_{2}}\left(\frac{t}{\epsilon}, k_{\ell}, k_{m}, k^{\prime}\right):=Q_{i_{\ell}, j_{1}}\left(\frac{t}{\epsilon}, k_{\ell}, k_{\ell}^{\prime}\right) Q_{i_{m}, j_{2}}\left(\frac{t}{\epsilon}, k_{m}, k_{m}^{\prime}\right)
$$

and $\mathbf{k}_{\ell, m}^{\prime}=\left(k_{1}^{\prime}, \ldots, k_{p}^{\prime}\right)$, where $k_{p}^{\prime}:=k_{p}$ for $p \neq \ell, m$ and $k_{\ell}^{\prime}:=k_{\ell}-k^{\prime}, k_{m}^{\prime}:=k_{m}+k^{\prime}$.

2) If the initial condition is from $\mathcal{M}\left(\mathbb{T}^{p}\right)$ then the solution also belongs to $\mathcal{M}\left(\mathbb{T}^{p}\right)$ and for any $t_{*}>0$

$$
M_{*}(T):=\sum_{\mathbf{i} \in\{-1,1\}^{p}} \sup _{\epsilon \in(0,1]} \sup _{t \in\left[0, t_{*}\right]}\left\|\hat{M}_{\mathbf{i}}^{(\epsilon)}(t)\right\|_{\mathrm{TV}}<+\infty
$$

Proof. The fact that $\hat{M}^{(\epsilon)}(t)$ is a solution of (5.10) follows by an application of Itô formula and equation (5.8). Since the operators appearing on the right hand side of the equation in question are uniformly Lipschitz, on any compact time interval, both in $H^{-m / p}\left(\mathbb{T}^{p}\right)$ and $\mathcal{M}\left(\mathbb{T}^{p}\right)$ the proof of uniqueness of solutions in these spaces is standard. Estimate (5.11) follows by an application of Gronwall's inequality.

\subsection{Asymptotics of even moments}

Let us now describe the limit moment equations. Assume that $p=2 n$ is even, then for any $1 \leq \ell<m \leq 2 n$ let $D_{\ell, m}:=\left[\mathbf{k} \in \mathbb{T}^{2 n}: k_{\ell}=-k_{m}\right]$. We define a bounded linear operator $\mathcal{R}_{\ell, m}: \mathcal{M}\left(\mathbb{T}^{2 n}\right) \rightarrow \mathcal{M}\left(\mathbb{T}^{2 n}\right)$ by

$$
\int_{\mathbb{T}^{2 n}} f d \mathcal{R}_{\ell, m} \nu:=\int_{\mathbb{T}} d k\left\{\int_{D_{\ell, m}} r^{2}\left(k, k-k_{\ell}^{\prime}\right) f\left(S\left(\mathbf{k}^{\prime}, k\right)\right) \nu\left(d \mathbf{k}^{\prime}\right)\right\}
$$

for any bounded, measurable $f: \mathbb{T}^{2 n} \rightarrow \mathbb{C}$ and $\nu \in \mathcal{M}\left(\mathbb{T}^{2 n}\right)$. We define $S: \mathbb{T}^{2 n+1} \rightarrow \mathbb{T}^{2 n}$ as follows: given $\mathbf{k}^{\prime}=\left(k_{1}^{\prime}, \ldots, k_{2 n}^{\prime}\right) \in \mathbb{T}^{2 n}$ and $k \in \mathbb{T}$ we let $\left(k_{1}, \ldots, k_{2 n}\right)=S\left(\mathbf{k}^{\prime}, k\right)$ if $k_{j}=k_{j}^{\prime}$ for $j \notin\{\ell, m\}$ and $k_{\ell}=k, k_{m}=-k$. 
Suppose that the components of the tensor $\hat{M}=\left[\hat{M}_{\mathbf{i}}\right]$ belong to $\mathcal{M}\left(\mathbb{T}^{2 n}\right)$. Similarly to part 1) of Proposition 5.2 we conclude that the initial value problem

$$
\begin{aligned}
& \frac{d}{d t} \hat{M}_{\mathbf{i}}(t)=-\frac{1}{4}\left(\sum_{\ell=1}^{2 n} \hat{\beta}\left(k_{\ell}\right)\right) \hat{M}_{\mathbf{i}}(t)+\sum_{1 \leq \ell<m \leq 2 n} \sum_{j= \pm 1} \mathcal{R}_{\ell, m} \hat{M}_{\mathbf{i}_{\ell, m}(j,-j)}(t), \\
& \hat{M}(0)=\hat{M}
\end{aligned}
$$

possesses a unique solution in $C\left([0,+\infty), \mathcal{M}\left(\mathbb{T}^{2 n}\right)\right)$.

Any partition of the set $\{1, \ldots, 2 n\}$ into a disjoint set of pairs is called a pairing. Define

$$
\mu(d \mathbf{k})=\sum_{\mathcal{F}} \prod_{(\ell, m) \in \mathcal{F}} \delta\left(k_{\ell}+k_{m}\right) d \mathbf{k},
$$

where $d \mathbf{k}=d k_{1} \ldots d k_{2 n}$ and the summation extends over all possible pairings of $\{1, \ldots, 2 n\}$. The measure is supported in $\mathbb{H}:=\bigcup_{\mathcal{F}} \mathbb{H}(\mathcal{F})$ where

$$
\mathbb{H}(\mathcal{F}):=\left[\mathbf{k}: k_{\ell}+k_{m}=0, \forall(\ell, m) \in \mathcal{F}\right] .
$$

Suppose that the components of the tensor $\rho(\mathbf{k})=\left[\rho_{\mathbf{i}}(\mathbf{k})\right], \mathbf{i} \in\{-1,1\}^{2 n}$ belong to $L^{1}(\mu)$. Consider the initial value problem

$$
\begin{aligned}
& \frac{d}{d t} \rho_{\mathbf{i}}(t, \mathbf{k})=-\frac{1}{4}\left(\sum_{\ell=1}^{2 n} \hat{\beta}\left(k_{\ell}\right)\right) \rho_{\mathbf{i}}(t, \mathbf{k}) \\
& +\sum_{1 \leq \ell<m \leq 2 n} \sum_{j= \pm 1} \int_{\mathbb{T}} r^{2}\left(k_{\ell}, k_{\ell}-k^{\prime}\right) 1_{D_{\ell, m}}(\mathbf{k}) \rho_{\mathbf{i}_{\ell, m}(j,-j)}\left(t, \mathbf{k}_{\ell, m}^{\prime}\right) d k^{\prime}, \\
& \rho_{\mathbf{i}}(0, \mathbf{k})=\rho_{\mathbf{i}}(\mathbf{k}), \mathbf{i} \in\{-1,1\}^{2 n},
\end{aligned}
$$

with $\mathbf{k}_{\ell, m}^{\prime}:=\left(k_{1}, \ldots, k_{\ell-1}, k^{\prime}, \ldots, k_{m-1},-k^{\prime}, \ldots, k_{2 n}\right)$. It is straightforward to conclude that the above system possesses a unique continuous solution $\rho(t, \mathbf{k})=\left[\rho_{\mathbf{i}}(t, \mathbf{k})\right]$ whose components belong to $L^{1}(\mu)$. The next proposition gives the convergence of even moments to the solution of (5.12).

Proposition 5.3 Suppose that all the components of the tensor $\left[\hat{M}_{\mathbf{i}}(d \mathbf{k})\right]$ are absolutely continuous with respect to $\mu$, i.e. $\hat{M}_{\mathbf{i}}(d \mathbf{k})=\rho_{\mathbf{i}}(\mathbf{k}) \mu(d \mathbf{k})$, and the dispersion relation satisfies hypothesis $\omega)$. Then, the following are true:

1) $\hat{M}_{\mathbf{i}}(t, d \mathbf{k})$ is absolutely continuous with respect to $\mu(d \mathbf{k})$ and

$$
\hat{M}_{\mathbf{i}}(t, d \mathbf{k})=\rho_{\mathbf{i}}(t, \mathbf{k}) \mu(d \mathbf{k}), \quad \forall \mathbf{i} \in\{-1,1\}^{2 n}
$$

where $\left\{\rho_{\mathbf{i}}(t), t \geq 0\right\}$ satisfy (5.13) .

2) For any $T>0$ there exists a constant $C>0$ such that

$$
\lim _{\epsilon \rightarrow 0+} \sum_{\mathbf{i} \in\{-1,1\}^{2 n}} \sup _{t \in\left[0, t_{*}\right]}\left\|\hat{M}_{\mathbf{i}}^{(\epsilon)}(t)-\hat{M}_{\mathbf{i}}(t)\right\|_{\mathrm{TV}}=0 .
$$


Proof. The conclusion of part 1) follows from uniqueness of solutions of (5.12) and (5.13), and the fact that the right hand side of (5.14) defines a solution of (5.12). From (5.10) and (5.12) we conclude that

$$
\begin{aligned}
& \left\|\hat{M}_{\mathbf{i}}^{(\epsilon)}(t)-\hat{M}_{\mathbf{i}}(t)\right\|_{\mathrm{TV}} \leq \sum_{\ell=1}^{2 n} \sum_{j= \pm 1} \int_{0}^{t}\left\|C_{i_{\ell}, j}\left(\frac{s}{\epsilon}\right)\left[\hat{M}_{\mathbf{i}_{\ell}(j)}^{(\epsilon)}(s)-\hat{M}_{\mathbf{i}_{\ell}(j)}(s)\right]\right\|_{\mathrm{TV}} d s \\
& +\sum_{1 \leq \ell<m \leq 2 n} \sum_{j_{1}, j_{2}= \pm 1} \int_{0}^{t}\left\|\mathcal{R}_{i_{\ell}, i_{m}}^{j_{1}, j_{2}}\left(\frac{s}{\epsilon}\right)\left[\hat{M}_{\mathbf{i}_{\ell, m}\left(j_{1}, j_{2}\right)}^{(\epsilon)}(s)-\hat{M}_{\mathbf{i}_{\ell, m}\left(j_{1}, j_{2}\right)}(s)\right]\right\|_{\mathrm{TV}} d s \\
& +\sum_{\ell=1}^{2 n} \sum_{j= \pm 1}\left|\int_{0}^{t} \int_{\mathbb{T}^{2 n}} E_{i_{\ell}, j}\left(\frac{s}{\epsilon}, k_{\ell}\right) \rho_{\mathbf{i}_{\ell}(j)}(s, \mathbf{k}) d s \mu(d \mathbf{k})\right| \\
& +\sum_{1 \leq \ell<m \leq 2 n} \sum_{j_{1}, j_{2}= \pm 1}\left|\int_{0}^{t} \int_{\mathbb{T}^{2 n+1}} \tilde{\mathcal{R}}_{i_{\ell}, i_{m}}^{j_{1}, j_{2}}\left(\frac{s}{\epsilon}, \mathbf{k}, k^{\prime}\right) \rho_{\mathbf{i}_{\ell, m}\left(j_{1}, j_{2}\right)}(s, \mathbf{k}) d s \mu(d \mathbf{k}) d k^{\prime}\right| .
\end{aligned}
$$

The matrix $\mathbf{E}(t, k)=\left[E_{p, q}(t, k)\right], p, q= \pm 1$ is given by

$$
\mathbf{E}(t, k):=\mathbf{C}(t, k)-(\hat{\beta}(k) / 4) \mathbf{I}_{2},
$$

where $\mathbf{I}_{2}$ is the $2 \times 2$ identity matrix. In addition,

$$
\tilde{\mathcal{R}}_{i_{\ell}, i_{m}}^{j_{1}, j_{2}}\left(\frac{s}{\epsilon}, \mathbf{k}, k^{\prime}\right):=\mathcal{R}_{i_{\ell}, i_{m}}^{j_{1}, j_{2}}\left(\frac{s}{\epsilon}, k_{\ell}, k_{m}, k^{\prime}\right)-\delta_{i_{\ell}}^{-i_{m}} \delta_{j_{1}}^{-j_{2}} r^{2}\left(k_{\ell}, k_{\ell}-k^{\prime}\right) 1_{D_{\ell, m}}(\mathbf{k}) .
$$

Denote the terms appearing on the right hand side of (5.16) by $I(t), I I(t), I I I(t)$ and $I V(t)$ respectively. It is easy to see that

$$
I(t)+I I(t) \leq C \int_{0}^{t} \sup _{\mathbf{i} \in\{-1,1\}^{2 n}}\left\|\hat{M}_{\mathbf{i}}^{(\epsilon)}(s)-\hat{M}_{\mathbf{i}}(s)\right\|_{\mathrm{TV}} d s
$$

for some constant $C>0$. To estimate the term $I I I$ we need to bound terms of the form

$$
\left|\int_{0}^{t} \int_{\mathbb{T}^{2 n}} \hat{\beta}\left(k_{\ell}\right) \exp \left\{2 i \omega\left(k_{\ell}\right) \frac{s}{\epsilon}\right\} \rho_{\mathbf{i}}(s, \mathbf{k}) d s \mu(d \mathbf{k})\right|
$$

for some $\ell$ and $\mathbf{i}$. Integrating by parts we obtain that the expression above can be bounded from above by

$$
\begin{aligned}
& \epsilon\left|\int_{\mathbb{T}^{2 n}} \frac{\hat{\beta}\left(k_{\ell}\right)}{2 i \omega\left(k_{\ell}\right)}\left[\exp \left\{2 i \omega\left(k_{\ell}\right) \frac{t}{\epsilon}\right\}-1\right] \rho_{\mathbf{i}}(t, \mathbf{k}) 1_{D_{\ell, m}}(\mathbf{k}) \mu(d \mathbf{k})\right| \\
& +\epsilon\left|\int_{0}^{t} \int_{\mathbb{T}^{2 n}} \frac{\hat{\beta}\left(k_{\ell}\right)}{2 i \omega\left(k_{\ell}\right)}\left[\exp \left\{2 i \omega\left(k_{\ell}\right) \frac{t}{\epsilon}\right\}-1\right] \frac{d}{d s} \rho_{\mathbf{i}}(s, \mathbf{k}) 1_{D_{\ell, m}}(\mathbf{k}) d s \mu(d \mathbf{k})\right| .
\end{aligned}
$$

The first term can be easily estimated by $C \epsilon$, due to the fact that $\sup _{k \in \mathbb{T}} \hat{\beta}(k) \omega^{-1}(k)<+\infty$. To estimate the second term, we use equation (5.13). As a result,, we conclude that for any $t_{*}>0$ we can find a constant $C\left(t_{*}\right)>0$ such that

$$
\sup _{t \in\left[0, t_{*}\right]} I I I(t) \leq C\left(t_{*}\right) \epsilon .
$$


Finally we show that

$$
\lim _{\epsilon \rightarrow 0+} \sup _{t \in\left[0, t_{*}\right]} I V(t)=0 .
$$

It implies the conclusion of part 2) of the proposition, via an application of the Gronwall's inequality.

We write $I V(t)=I_{1}(t)+I_{2}(t)$, where the terms $I_{i}(t), i=1,2$ correspond to the integration over $D_{\ell, m}$ and its complement. In the latter case, we have to deal with terms of the form

$$
\begin{aligned}
& \mid \int_{0}^{t} \int_{\mathbb{T}^{2 n+1}} 1_{\left[k_{\ell} \neq-k_{m}\right]} r\left(k_{\ell}, k^{\prime}\right) r\left(k_{m},-k^{\prime}\right) \rho_{\mathbf{i}}(s, \mathbf{k}) \\
& \times \prod_{j=1}^{2} \exp \left\{i \sigma_{1}^{(j)}\left[\omega\left(k_{\ell}^{(j)}\right)+\sigma_{2}^{(j)} \omega\left(k_{\ell}^{(j)}+(-1)^{j} k^{\prime}\right)\right] \frac{s}{\epsilon}\right\} d s \mu(d \mathbf{k}) d k^{\prime} \mid
\end{aligned}
$$

for some $\mathbf{i} \in\{-1,1\}^{2 n}, \sigma_{p}^{(j)} \in\{-1,1\}$. Here $k_{\ell}^{(1)}=k_{\ell}$ and $k_{\ell}^{(2)}=k_{m}$. Using integration by parts over the $s$ variable we can estimate the supremum of the above expression over $t \in\left[0, t_{*}\right]$ by the sum of

$$
\begin{aligned}
& I_{\epsilon}:=\int_{\mathbb{T}^{2 n+1}} \mu(d \mathbf{k}) d k^{\prime} 1_{\left[k_{\ell} \neq-k_{m}\right]}\left|r\left(k_{\ell}, k^{\prime}\right) r\left(k_{m},-k^{\prime}\right)\right| \sup _{t \in\left[0, t_{*}\right]}\left|\rho_{\mathbf{i}}(t, \mathbf{k})\right| \\
& \times \epsilon\left|\sum_{j=1}^{2} \sigma_{1}^{(j)}\left[\omega\left(k_{\ell}^{(j)}\right)+\sigma_{2}^{(j)} \omega\left(k_{\ell}^{(j)}+(-1)^{j} k^{\prime}\right)\right]\right|^{-1} \\
& \times \sup _{t \in\left[0, t_{*}\right]} \prod_{j=1}^{2}\left|\exp \left\{i \sigma_{1}^{(j)}\left[\omega\left(k_{\ell}^{(j)}\right)+s i_{2}^{(j)} \omega\left(k_{\ell}^{(j)}+(-1)^{j} k^{\prime}\right)\right] \frac{t}{\epsilon}\right\}-1\right|,
\end{aligned}
$$

and

$$
\begin{aligned}
& J_{\epsilon}:=\int_{0}^{T} d s \mid \int_{\mathbb{T}^{2 n+1}} \mu(d \mathbf{k}) d k^{\prime} 1_{\left[k_{\ell} \neq-k_{m}\right]} r\left(k_{\ell}, k^{\prime}\right) r\left(k_{m},-k^{\prime}\right) \frac{d}{d s} \rho_{\mathbf{i}}(s, \mathbf{k}) \\
& \times \epsilon\left\{\sum_{j=1}^{2} \sigma_{1}^{(j)}\left[\omega\left(k_{\ell}^{(j)}\right)+\sigma_{2}^{(j)} \omega\left(k_{\ell}^{(j)}+(-1)^{j} k^{\prime}\right)\right]\right\}^{-1} \\
& \times \prod_{j=1}^{2}\left\{\exp \left\{i \sigma_{1}^{(j)}\left[\omega\left(k_{\ell}^{(j)}\right)+\sigma_{2}^{(j)} \omega\left(k_{\ell}^{(j)}+(-1)^{j} k^{\prime}\right)\right] \frac{s}{\epsilon}\right\}-1\right\} \mid .
\end{aligned}
$$

Using (5.13) and Gronwall's inequality, we conclude that

$$
\int_{\mathbb{T}^{2 n}} \sup _{t \in\left[0, t_{*}\right]}\left|\rho_{\mathbf{i}}(t, \mathbf{k})\right| d \mathbf{k}<+\infty .
$$

Using condition $\omega$ ) we conclude therefore, by virtue of Lebesgue dominated convergence theorem, that $\lim _{\epsilon \rightarrow 0+} I_{\epsilon}=0$. Likewise, after substituting for $\rho_{\mathbf{i}}^{\prime}(s, \mathbf{k})$ from (5.13), we conclude that 
$\lim _{\epsilon \rightarrow 0+} J_{\epsilon}=0$. Part 2) of the proposition follows then from another application of Gronwall's inequality. Summarizing, we have shown so far that

$$
\lim _{\epsilon \rightarrow 0+} \sup _{t \in\left[0, t_{*}\right]} I V_{2}(t)=0 .
$$

We are left therefore with estimates of the term

$$
\begin{aligned}
& V_{1}(t):=\sum_{1 \leq \ell<m \leq 2 n} \sum_{j_{1}, j_{2}= \pm 1} \mid \int_{0}^{t} \int_{\mathbb{T}^{2 n+1}} 1_{D_{\ell, m}}(\mathbf{k}) \\
& \times \tilde{\mathcal{R}}_{i_{\ell}, i_{m}}^{j_{1}, j_{2}}\left(\frac{s}{\epsilon}, \mathbf{k}, k^{\prime}\right) \rho_{\mathbf{i}_{\ell, m}\left(j_{1}, j_{2}\right)}(s, \mathbf{k}) d s \mu(d \mathbf{k}) d k^{\prime} \mid .
\end{aligned}
$$

The non-vanishing terms appearing in the above sum are of the form

$$
\left|\int_{0}^{t} \int_{\mathbb{T}^{2 n+1}} r^{2}\left(k_{\ell}, k_{\ell}-k^{\prime}\right) 1_{D_{\ell, m}}(\mathbf{k}) \prod_{j=1}^{2} \exp \left\{i \sigma_{1}^{(j)}\left[\omega\left(k_{\ell}\right)+\sigma_{2}^{(j)} \omega\left(k_{\ell}-k^{\prime}\right)\right] \frac{s}{\epsilon}\right\} d s \mu(d \mathbf{k}) d k^{\prime}\right|,
$$

with $\left(\sigma_{1}^{(1)}, \sigma_{2}^{(1)}\right) \neq-\left(\sigma_{1}^{(2)}, \sigma_{2}^{(2)}\right)$ and $\sigma_{p}^{(j)} \in\{-1,1\}$. To these terms we can apply the integration by parts argument as before, to conclude that

$$
\lim _{\epsilon \rightarrow 0+} \sup _{t \in\left[0, t_{*}\right]} I V_{1}(t)=0 .
$$

Summarizing, we have shown that (5.19) holds, and the proof of part 2 of the proposition is therefore complete.

\subsection{Proof of Theorem 3.3}

In this section, and in this section only, we make use of the assumption that $\hat{\psi}$ is Gaussian. We show that the limiting measure for $\tilde{Q}_{\epsilon}$, as $\epsilon \rightarrow 0+$, coincides with the law $\tilde{Q}$ of the process given (3.17) by proving that for any $N \geq 1,0 \leq t_{1}<\ldots<t_{N}$, any non-negative integers $\ell_{j}, m_{j}$, test functions $f_{j}, g_{j} \in H^{m}(\mathbb{T}), j=1, \ldots, N$ we have

$$
\lim _{\epsilon \rightarrow 0+} \mathbb{E}\left[\prod_{j=1}^{N}\left[\left\langle\tilde{\psi}^{(\epsilon)}\left(t_{j}\right), f_{j}\right\rangle^{\ell_{j}}\left(\left\langle\tilde{\psi}^{(\epsilon)}\left(t_{j}\right), g_{j}\right\rangle^{*}\right)^{m_{j}}\right]\right]=\mathbb{E}\left[\prod_{j=1}^{N}\left[\left\langle\bar{\psi}\left(t_{j}\right), f_{j}\right\rangle^{\ell_{j}}\left(\left\langle\bar{\psi}\left(t_{j}\right), g_{j}\right\rangle^{*}\right)^{m_{j}}\right]\right] .
$$

To simplify the notation, we prove (5.23) only in the case $N=1$. The general case can be handled in the same manner, using Markov property of the process $\left\{\tilde{\psi}^{(\epsilon)}(t), t \geq 0\right\}$, at the expense of some additional complications in the notation. We recall (see Section 3.2) that the initial data $\{\hat{\psi}(k), k \in \mathbb{T}\}$ is a $\delta$-correlated Gaussian random field given by (3.15). Therefore, for the odd moments we have

$$
\hat{M}_{\mathbf{i}}^{(\epsilon)}(0)=0, \quad \forall \mathbf{i} \in\{-1,1\}^{2 n-1},
$$

where $n \geq 1$ is an integer. By uniqueness of solutions of (5.10) we conclude that in this case $\hat{M}^{(\epsilon)}(t) \equiv 0$ for all $t \geq 0$. When $\mathbf{i} \in\{-1,1\}^{2 n}$ we can use the conclusion (5.15) of Proposition 5.3. Define

$$
\bar{M}^{(2 n)}(t):=\left[\bar{M}_{\mathbf{i}}^{(2 n)}(t)\right], \quad \mathbf{i}=\left(i_{1}, \ldots, i_{2 n}\right) \in\{-1,1\}^{2 n},
$$


where

$$
\bar{M}_{\mathbf{i}}^{(2 n)}(t)=\mathbb{E}\left[\bar{\psi}_{i_{1}}(t) \otimes \ldots \otimes \bar{\psi}_{i_{2 n}}(t)\right]
$$

and $\bar{\psi}_{1}(t)=\bar{\psi}(t)$ is the solution of (3.17) and $\bar{\psi}_{-1}(t, k)=\bar{\psi}^{*}(t,-k)$. The conclusion of Theorem 3.3 will follow provided that we show that $\bar{M}^{(2 n)}(t)$, satisfies (5.12). Note that for $n=1$ we obtain that

$$
\bar{M}_{i_{1}, i_{2}}^{(2)}(t, d \mathbf{k})=\delta_{i_{1},-i_{2}} \overline{\mathcal{E}}\left(t, k_{1}\right) \delta\left(k_{1}+k_{2}\right) d k_{1} d k_{2} .
$$

From (3.17) and Itô formula we conclude that

$$
\begin{aligned}
& \frac{d}{d t} \bar{M}_{\mathbf{i}}^{(2 n)}(t)=-\frac{1}{4}\left(\sum_{\ell=1}^{2 n} \hat{\beta}\left(k_{\ell}\right)\right) \bar{M}_{\mathbf{i}}^{(2 n)}(t)-\sum_{1 \leq \ell<m \leq 2 n} \mathcal{R}\left(t, k_{\ell}\right) \bar{M}_{\mathbf{i}_{\ell, m}}^{(2 n-2)}(t) \otimes_{\ell, m} \Delta, \\
& \bar{M}(0)=\hat{M} .
\end{aligned}
$$

Here $\bar{M}_{\mathbf{i}_{\ell, m}}^{(2 n-2)}(t)$ is the $2 n-2$-nd order moment obtained from $\bar{M}_{\mathbf{i}}^{(2 n)}(t)$ by omitting $\bar{\psi}_{i_{\ell}}(t)$ and $\bar{\psi}_{i_{m}}(t)$ and for any measure $\nu$ on $\mathbb{T}^{2 n-2}, 1 \leq \ell<m \leq 2 n$ we denote by $\nu \otimes_{\ell, m} \Delta$ a measure on $\mathbb{T}^{2 n}$ given by

$$
\int_{\mathbb{T}^{2 n}} f d\left(\nu \otimes_{\ell, m} \Delta\right)=\int_{\mathbb{T}^{2 n-2}} d \mathbf{k} \int_{\mathbb{T}} d k f\left(k_{1}, \ldots, k_{\ell-1}, k, \ldots, k_{m-1},-k, \ldots, k_{2 n-2}\right)
$$

for all $f \in C\left(\mathbb{T}^{2 n}\right)$. Since

$$
\begin{aligned}
& \mathcal{R}\left(t, k_{\ell}\right)=\int_{\mathbb{T}} R\left(k_{\ell}, k^{\prime}\right) \overline{\mathcal{E}}\left(t, k^{\prime}\right) d k^{\prime}=\int_{\mathbb{T}}\left[r^{2}\left(k_{\ell}, k_{\ell}-k^{\prime}\right)+r^{2}\left(k_{\ell}, k_{\ell}+k^{\prime}\right)\right] \overline{\mathcal{E}}\left(t, k^{\prime}\right) d k^{\prime} \\
& =\sum_{j= \pm 1} \int_{\mathbb{T}^{2}} r^{2}\left(k_{\ell}, k_{\ell}-k^{\prime}\right) \mathbb{E}\left[\bar{\psi}_{j}\left(t, k^{\prime}\right) \otimes \bar{\psi}_{-j}\left(t, k^{\prime \prime}\right)\right] d k^{\prime} d k^{\prime \prime}
\end{aligned}
$$

and $\left(\bar{\psi}_{i_{1}}(t), \ldots, \bar{\psi}_{i_{2 n}}(t)\right)$ is jointly Gaussian, we infer that the last term on the right hand side of the first equation in (5.24) equals the last term on the right hand side of the first equation of (5.12). Thus the conclusion of Theorem 3.3 has been shown.

\section{A Proof of Proposition 2.1}

To prove the proposition we verify that for any $T>0$

$$
t \mapsto \mathcal{A}[t, \cdot] \quad \text { is Lipschitz on } H^{m}(\mathbb{T}),
$$

uniformly in $t \in[0, T]$ and $\tilde{Q}[t, g]: L^{2}(\mathbb{T}) \rightarrow H^{m}(\mathbb{T})$, given by (2.20) is Hilbert-Schmidt for any $g \in H^{m}(\mathbb{T})$ and its respective Hilbert-Schmidt norm satisfies

$$
\sup _{t \in[0, T]}\left\|\tilde{Q}\left[t, g_{1}\right]-\tilde{Q}\left[t, g_{2}\right]\right\|_{(H S)_{m}} \leq C\left\|g_{1}-g_{2}\right\|_{H^{m}(\mathbb{T})}, \quad \forall g_{1}, g_{2} \in H^{m}(\mathbb{T})
$$

for some $C>0$. The conclusion of the lemma then follows from [7, Theorem 7.4, p. 186 . 
Since $\beta_{x} \neq 0$ only for $|x| \leq 2$, see (2.2), to prove (A.1) it suffices only to show that there exists $C>0$ such that

$$
\sup _{t \in[0, T]}\|f(t)\|_{H^{m}(\mathbb{T})} \leq C\|f\|_{H^{m}(\mathbb{T})}, \quad \forall f \in H^{m}(\mathbb{T}),
$$

with $f(t):=\exp \{2 i \omega(k) t\} f(k)$. Dispersion relation $\omega(\cdot)$ given by (2.10) is bounded with its all derivatives on $\mathbb{T} \backslash\{0\}$. In addition $\omega^{\prime}(0-)$ and $\omega^{\prime}(0+)$ exist. Therefore

$$
\omega_{*}:=\sup _{t \in[0, T], x \in \mathbb{Z}}\left(1+x^{2}\right)\left|\gamma_{x}(t)\right|<+\infty,
$$

where

$$
\gamma_{x}(t):=\int_{\mathbb{T}} \exp \{2 i \omega(k) t\} e_{x}^{*}(k) d k
$$

Note that

$$
1+y^{2} \leq \sup _{x} \frac{1+x^{2}}{1+(x-y)^{2}} \leq 2\left(1+y^{2}\right)
$$

Assume first that $m \geq 0$. We can write then

$$
\begin{aligned}
& \|f(t)\|_{H^{m}(\mathbb{T})}^{2}=\sum_{x \in \mathbb{Z}}\left(1+x^{2}\right)^{m}\left|\check{f}_{x}(t)\right|^{2}=\sum_{x \in \mathbb{Z}}\left(1+x^{2}\right)^{m}\left|\sum_{y \in \mathbb{Z}} \check{f}_{x-y} \gamma_{y}(t)\right|^{2} \\
& =\sum_{x \in \mathbb{Z}}\left|\sum_{y \in \mathbb{Z}}\left(1+(x-y)^{2}\right)^{m / 2} \check{f}_{x-y} \frac{\left(1+x^{2}\right)^{m / 2} \gamma_{y}(t)}{\left(1+(x-y)^{2}\right)^{m / 2}}\right|^{2} .
\end{aligned}
$$

Using (A.4) together with (A.5) we can we can estimate the utmost right hand side of (A.6) by

$$
2 \omega_{*}^{2} \sum_{x \in \mathbb{Z}}\left\{\sum_{y \in \mathbb{Z}}\left(1+(x-y)^{2}\right)^{m / 2}\left|\check{f}_{x-y}\right|\left(1+y^{2}\right)^{m / 2-1}\right\}^{2} .
$$

Using Young's inequality $\|f * g\|_{\ell^{r}} \leq\|f\|_{\ell^{p}}\|g\|_{\ell^{q}}$, where $1+r^{-1}=p^{-1}+q^{-1}$, (with $r=p=2$, $q=1$ ) we can bound this expression by

$$
C\left\{\sum_{x \in \mathbb{Z}}\left(1+x^{2}\right)^{m}\left|\check{f}_{x}\right|^{2}\right\}\left\{\sum_{y \in \mathbb{Z}}\left(1+y^{2}\right)^{m / 2-1}\right\}^{2}
$$

for some constant $C>0$. Summarizing we have shown that

$$
\|f(t)\|_{H^{m}(\mathbb{T})}^{2} \leq 2 \omega_{*}^{2}\|f\|_{H^{m}(\mathbb{T})}^{2}\left\{\sum_{y}\left(1+y^{2}\right)^{m / 2-1}\right\}^{2},
$$

which proves (A.3), provided $0 \leq m<1$.

If, on the other hand, $m<0$ we can write

$$
\sum_{x \in \mathbb{Z}}\left(1+x^{2}\right)^{m}\left|\check{f}_{x}(t)\right|^{2} \leq \omega_{*}^{2} \sum_{x \in \mathbb{Z}}\left(1+x^{2}\right)^{m}\left[\sum_{y \in \mathbb{Z}}\left(1+y^{2}\right)^{-1}\left|\check{f}_{x-y}\right|\right]^{2} .
$$


By Cauchy-Schwartz inequality for any $\gamma>1 / 2$ the right hand side can be estimated by

$$
\omega_{*}^{2} \sum_{x \in \mathbb{Z}}\left(1+x^{2}\right)^{m}\left[\sum_{y \in \mathbb{Z}}\left(1+y^{2}\right)^{-\gamma}\right]\left[\sum_{y \in \mathbb{Z}}\left(1+(x-y)^{2}\right)^{-(2-\gamma)}\left|\check{f}_{y}\right|^{2}\right] .
$$

We use the following elementary inequality: for any $\kappa>1 / 2$ there exists a constant $C>0$ such that

$$
\sum_{x \in \mathbb{Z}}\left(1+x^{2}\right)^{m}\left(1+(x-y)^{2}\right)^{-\kappa} \leq C\left(1+y^{2}\right)^{m \vee(-\kappa)}, \quad \forall y \in \mathbb{Z} .
$$

Let $\kappa:=2-\gamma$ and $\gamma \in(1 / 2,3 / 2)$. We conclude from the above estimate that the expression in (A.7) is less than, or equal to $C\|f\|_{H^{m}(\mathbb{T})}^{2}$, provided that $2+m>\gamma$, which is possible as long as $m>-3 / 2$.

To show (A.2) it suffices to prove that for any functions $\phi_{1}, \phi_{2}$ that are finite combinations of the vectors from the base $\left(e_{x}\right)$ and $T>0$ there exists a constant $C>0$ such that

$$
\begin{aligned}
& \sup _{t \in[0, T]} \sum_{x, y}\left(1+y^{2}\right)^{m}\left|\int_{\mathbb{T}^{2}} \phi_{1}(k) \phi_{2}\left(k-k^{\prime}\right) g\left(k-k^{\prime}\right) e_{x}\left(k^{\prime}\right) e_{y}^{*}(k) \exp \left\{i\left[\omega(k)-\sigma \omega\left(k-k^{\prime}\right)\right] t\right\} d k d k^{\prime}\right|^{2} \\
& \leq C\|g\|_{H^{m}(\mathbb{T})}^{2}, \quad \forall g \in H^{m}(\mathbb{T}), \sigma= \pm 1 .
\end{aligned}
$$

The expression on the left hand side of (A.9) can be rewritten in the form

$$
\sum_{x, y}\left(1+y^{2}\right)^{m}\left|\psi_{y-x}^{(1)}(t) \psi_{x}^{(2)}(t)\right|^{2}
$$

where

$$
\psi_{x}^{(1)}(t):=\int_{\mathbb{T}} \phi_{1}(k) e_{x}^{*}(k) \exp \{i \omega(k) t\} d k
$$

and

$$
\psi_{x}^{(2)}(t):=\int_{\mathbb{T}} \phi_{2}(k) g(k) e_{x}^{*}(k) \exp \{-i \sigma \omega(k) t\} d k .
$$

As a consequence of (A.3) for any $T>0$ there exists $C>0$ such that

$$
\sup _{t \in[0, T]} \sum_{x}\left(1+x^{2}\right)^{m}\left|\psi_{x}^{(2)}(t)\right|^{2} \leq C\|g\|_{H^{m}(\mathbb{T})}^{2}, \quad \forall g \in H^{m}(\mathbb{T}) .
$$

We also have $\sup _{t \in[0, T]}\left(1+x^{2}\right)\left|\psi_{x}^{(1)}(t)\right|<+\infty$. The expression in (A.10) can be rewritten as

$$
\begin{aligned}
& \sum_{x, z}\left(1+(z+x)^{2}\right)^{m}\left|\psi_{z}^{(1)}(t) \psi_{x}^{(2)}(t)\right|^{2} \\
& =\sum_{x, z} \frac{\left(1+(z+x)^{2}\right)^{m}}{\left(1+x^{2}\right)^{m}}\left(1+x^{2}\right)^{m}\left|\psi_{z}^{(1)}(t) \psi_{x}^{(2)}(t)\right|^{2} .
\end{aligned}
$$


Suppose that $m \geq 0$ then the right hand side can be estimated by

$$
\begin{aligned}
& 2^{m} \sum_{x, z}\left(1+z^{2}\right)^{m}\left(1+x^{2}\right)^{m}\left|\psi_{z}^{(1)}(t) \psi_{x}^{(2)}(t)\right|^{2} \\
& =2^{m}\left(\sum_{z}\left(1+z^{2}\right)^{m}\left|\psi_{z}^{(1)}(t)\right|^{2}\right)\left(\sum_{x}\left(1+x^{2}\right)^{m}\left|\psi_{x}^{(2)}(t)\right|^{2}\right) \\
& \leq C\left(\sum_{z}\left(1+z^{2}\right)^{m-2}\right)\|g\|_{H^{m}(\mathbb{T})}^{2},
\end{aligned}
$$

which proves (A.2), provided that $0 \leq m<3 / 2$.

If, on the other hand, $m<0$ the left hand side of (A.11) can be estimated by

$$
\begin{aligned}
& C \sum_{x, z}\left(1+(z+x)^{2}\right)^{m}\left(1+z^{2}\right)^{-2}\left|\psi_{x}^{(2)}(t)\right|^{2} \\
& \leq C_{1} \sum_{x}\left(1+x^{2}\right)^{m \vee(-2)}\left|\psi_{x}^{(2)}(t)\right|^{2} \leq C_{2}\|g\|_{H^{m}(\mathbb{T})}^{2},
\end{aligned}
$$

provided that $m>-2$.

\section{References}

[1] G. Bal, T. Komorowski and L. Ryzhik, Asymptotics of the solutions of the random Schrödinger equation. Arch. Ration. Mech. Anal. 200, 2011, 613-664.

[2] G. Basile, C. Bernardin, and S. Olla, A momentum conserving model with anomalous thermal conductivity in low dimension, Phys. Rev. Lett. 96, 2006, 204303.

[3] G. Basile, C. Bernardin, and S. Olla, Thermal conductivity for a momentum conserving model, Comm.Math.Phys., 287, 2009, 67-98.

[4] G. Basile, S. Olla, H. Spohn, Energy transport in stochastically perturbed lattice dynamics, Arch.Rat.Mech., 195, 2009, 171-203.

[5] C. Bernardin, S. Olla, Fourier's law for a microscopic model of heat conduction, Jour. Stat. Phys., 118, 2005, 271-289.

[6] P. Billingsley, Convergence of Probability Measures, New York: Wiley 1968

[7] Da Prato, G., Zabczyk, J., Stochastic Equations in Infinite Dimensions, Cambridge Univ. Press, (1992).

[8] Gilbarg, D. and Trudinger, N.S., Elliptic Partial Differential Equations of Second Order, Springer Verlag, Berlin, 1998.

[9] Helland, I, S., Central limit theorems for martingales with discrete, or continuous time, Scan. J. Statist., 9, 1982, 79-94. 
[10] A. Jakubowski, On the Skorokhod topology, Annales de l'I.H.P., Section B, 22, 1986, 263-285.

[11] H. Kesten, G. C. Papanicolaou, A limit theorem for turbulent diffusion, Commun. Math. Phys. 65, 1979, 97-128.

[12] T. Komorowski, M. Jara, and S. Olla, Limit theorems for a additive functionals of a Markov chain, Annals of Applied Probability, 19, 2009, 2270-2300.

[13] T. Komorowski, Ł. Stȩpień, Long time, large scale limit of the Wigner transform for a system of linear oscillators in one dimension, Journ. Stat. Phys., 2012, 148, 1-37.

[14] S. Lepri, R. Livi, A. Politi, Thermal conduction in classical low-dimensional lattices, Phys. Rep. 377, 2003, 1-80.

[15] J. Lukkarinen and H. Spohn, Kinetic limit for wave propagation in a random medium, Arch. Rat. Mech. Anal. 183, 2007, 93-162.

[16] A. Mielke, Macroscopic behavior of microscopic oscillations in harmonic lattices via Wigner-Husimi transforms, Arch. Rat. Mech. Anal. 181, 2006, 401-448.

[17] I. Mitoma, On the sample continuity of $\mathcal{S}^{\prime}$ processes, J. Math. Soc. Japan, 35, 1983, 629636.

[18] H. Spohn, The phonon Boltzmann equation, properties and link to weakly anharmonic lattice dynamics, J. Stat. Phys. 124, 2006, 1041-1104.

[19] Stroock, D. W.; Varadhan, S. R. Srinivasa, Multidimensional diffusion processes. Reprint of the 1997 edition. Classics in Mathematics. Springer-Verlag, Berlin, 2006.

[20] Zakharov, V. E., L'vov, V. S., Falkovich, G., Kolmogorov spectral of turbulence I. Wave turbulence., Springer 1992. 